\title{
Genetic Strain and Sex Differences in a Hyperoxia-Induced Mouse Model of Varying Severity of Bronchopulmonary Dysplasia
}

\author{
Sean Leary, ${ }^{*}$ Pragnya Das, ${ }^{*}$ Devasena Ponnalagu ${ }^{\dagger}$ Harpreet Singh, ${ }^{\dagger}$ and Vineet Bhandari ${ }^{* \sharp}$
}

From the Departments of Pediatrics* and Pharmacology, Physiology and Medicine, ${ }^{\dagger}$ Drexel University College of Medicine, Philadelphia; and the Division of Neonatology, ${ }^{\ddagger}$ St. Christopher's Hospital for Children, Philadelphia, Pennsylvania

\author{
Accepted for publication \\ January 24, 2019. \\ Address correspondence to \\ Vineet Bhandari, M.D., D.M., \\ Drexel University College of \\ Medicine, St. Christopher's \\ Hospital for Children, 160 East \\ Erie Avenue, Philadelphia, PA \\ 19134. E-mail: vineet. \\ bhandari@drexel.edu.
}

\begin{abstract}
Bronchopulmonary dysplasia (BPD) is a disease prevalent in preterm babies with a need for supplemental oxygen, resulting in impaired lung development and dysregulated vascularization. Epidemiologic studies have shown that males are more prone to BPD and have a delayed recovery compared with females, for reasons unknown. Herein, we tried to recapitulate mild, moderate, and severe BPD, using two different strains of mice, in males and females: CD1 (outbred) and C57BL/6 (inbred). Aside from higher body weight in the CD1 strain, there were no other gross morphologic differences with respect to alveolar development between the two strains. With respect to lung morphology after oxygen exposure, females had less injury with better preservation of alveolar chord length and decreased alveolar protein leak and inflammatory cells in the bronchoalveolar lavage fluid. In addition, housekeeping genes, which are routinely used as loading controls, were expressed differently in males and females. In the BPD mouse model, gonadotropin-releasing hormone was increased in females compared with males. Specific miRNAs (miR-146 and miR-34a) were expressed differently in the sexes. In the severe BPD mouse model, administering miR-146 mimic to males attenuated lung damage, whereas administering miR-146 inhibitor to females increased pulmonary injury. (Am J Pathol 2019, 189: 999-1014; https://doi.org/ 10.1016/j.ajpath.2019.01.014)
\end{abstract}

It has become increasingly recognized that genetic background can result in a different phenotype when the same genetic manipulation, treatment regimen, or environmental exposure is performed. ${ }^{1}$ Different strains may exhibit distinct levels of gene expression, although all mouse strains contain the same collection of genes. ${ }^{1,2}$ In addition, the same genetic alteration results in profoundly different phenotypes when present on different strain backgrounds, influencing various physiological and pathologic parameters studied. Whitehead et $\mathrm{al}^{3}$ studied the effect of oxygen injury on nine genetically diverse inbred strains after an acute exposure to hyperoxia and found that the response to $\mathrm{O}_{2}$ depends, in part, on the genetic background. It has also been previously suggested that outbred mouse strains should be limited in their use in research because of larger phenotypic variability and unknown genetic background, ${ }^{4}$ but there was no evidence to show that outbred strains show a lot of variability in disease models. Investigators have reported on three strains of mice (C57B1/6J, 129/J, and C3H/HeJ), ranging from sensitive to resistant, regarding their response to hyperoxia. $^{5}$ Others have used a variety of mice strains (BALB/c, $\mathrm{FVB} / \mathrm{n}, \mathrm{C} 3 \mathrm{H}$, and $129 \mathrm{SV}$ ) for neonatal hyperoxia studies. ${ }^{6} \mathrm{We}$ compared C57BL/6 (inbred) with CD1 (outbred) strains because, although the former is a well-established neonatal model for hyperoxia-induced injury, the latter has not been studied in the context of bronchopulmonary dysplasia (BPD), to the best of our knowledge.

There are certain diseases that are characteristic of one specific sex, whereas there may be others that are more common in one sex. In many instances, one sex responds to

Supported by the Department of Pediatrics and Drexel University funds 270926 (V.B.) and 282656 (V.B.), NIH grant R01-HL133050-01 (H.S.), and American Heart Association grant-in-aid 16GRNT29430000 (H.S.).

S.L. and P.D. contributed equally to this work.

Disclosures: None declared.

Current address of D.P. and H.S., Department of Physiology and Cell Biology, The Ohio State University, Columbus, $\mathrm{OH}$. 
the drugs more effectively than the other. ${ }^{7}$ Similarly, in the laboratory, researchers have a preference of working with one sex that is more specific to their area of research. Problems may arise when there is a discrepancy in the results because of sex differences. Most biomedical research is conducted with a preferential bias toward the male sex. As a result, most animal models of disease-related phenotypes remain poorly studied regarding sex differences. ${ }^{8}$ To raise awareness of this critical issue, the NIH stressed the importance of public health in studying sex differences in all disease models that are submitted as research grants to the NIH. ${ }^{9}$ Sex-specific differences exist in the development and the severity of BPD. ${ }^{10,11}$ In human studies, males born before the age of 26 weeks have shown a higher incidence of BPD and higher neonatal and infant mortality rates than females, after adjusting for confounding variables. ${ }^{10,12}$

Considering sex differences in all studies throughout the entire biomedical research pipeline is necessary to adequately inform clinical research and improve health outcomes. ${ }^{13}$ Hence, the goal of this study was to characterize the hyperoxia-induced BPD phenotype, in two different mouse strains, under varying oxygen exposures in an attempt to mimic mild $\left(40 \% \mathrm{O}_{2}\right)$, moderate $\left(60 \% \mathrm{O}_{2}\right)$, and severe $\left(100 \% \mathrm{O}_{2}\right) \mathrm{BPD}$, in males and females. Although no animal model can perfectly model the varying severities of human disease, we are able to show gradations of severity of lung pathology using the three different concentrations of oxygen noted above, as has been reported by others. ${ }^{14,15}$

\section{Materials and Methods}

\section{Animal Approval}

Wild-type CD1 and C57BL/6 mice were obtained from the Jackson Laboratory (Bar Harbor, ME). All animal work was approved by the Institutional Animal Care and Use Committee of Drexel University College of Medicine (Philadelphia, PA).

\section{Mouse Model of BPD}

Newborn pups of both strains were exposed to hyperoxia in an airtight Plexiglas container $(55 \times 40 \times 50 \mathrm{~cm})$ (BioSpherix, Parish, NY), as previously described. ${ }^{16,17}$ Exposure to supplemental oxygen began either immediately or within 8 hours after birth [designated as postnatal day (PN) 1] and was continued through PN4 (saccular stage of lung development), after which the animals were recovered in room air (RA) until PN14 (alveolar stage of lung development). Oxygen concentrations during the first 4 days of life included $40 \%, 60 \%$, and $100 \%$ in an effort to emulate mild, moderate, and severe BPD, respectively. Lactating dams were alternated between hyperoxia and RA to prevent oxygen toxicity in the adult mice during the 4 days of hyperoxia treatment. Litter size was limited to six to eight pups per dam, in both strains, to control for the effect of litter size on nutrition and growth. Pups were sacrificed on PN14. Throughout the experiment, free access to food and water was given, and oxygen concentrations were continually monitored.

\section{Bronchoalveolar Lavage}

Pups were sacrificed on PN14, and the trachea was cannulated with a small-caliber needle. Two volumes of $300 \mu \mathrm{L}$ of cold $1 \times$ phosphate-buffered saline were instilled, gently aspirated, and pooled. Samples were centrifuged at $1000 \times g$ for 10 minutes at $4{ }^{\circ} \mathrm{C}$. The supernatant was collected, and total protein was quantified using the Pierce BCA Protein Assay Kit (Fisher Scientific Co, Houston, TX). The cell pellet was dissolved in $200 \mu \mathrm{L} 1 \times$ phosphate-buffered saline and cytospun for differential and total cell count using the HEMA differential stain (Fisher Scientific, Kalamazoo, MI).

\section{Histology}

Both the RA control and BPD mice were anesthetized (using a cocktail of xylazine-ketamine), and tracheas were cannulated by instilling phosphate-buffered saline endotracheally at $25 \mathrm{~cm} \mathrm{H}_{2} \mathrm{O}$ pressure for 15 minutes. The trachea was tied, and lung and heart tissues were harvested after perfusion and fixed overnight in $4 \%$ paraformaldehyde. Fixed tissues were then washed in fresh phosphate-buffered saline, dehydrated using $70 \%$ ethanol, cleared, and embedded in paraffin to be stained with hematoxylin and eosin (H\&E), as previously described. ${ }^{16,17}$

\section{Lung, Heart, and Vascular Morphometry}

Left-lobe lung sections ( $5 \mu \mathrm{m}$ thick) were stained with H\&E. Multiple randomly chosen areas (at least 10 areas) from each section were imaged using $\times 100$ total magnification. Sections with large airways or blood vessels were excluded. Mean chord length of the airspace was estimated, as previously described, ${ }^{16}$ using ImageJ version 1.45 (NIH, Bethesda, MD; http://imagej.nih.gov/ij).

For measuring the right and left ventricular thickness, crosssections of whole hearts at the level of the atrial-ventricle junction were analyzed on H\&E-stained sections ( $5 \mu \mathrm{m}$ thick). The Fulton index was measured by calculating the ratio between the thickness of the right ventricle (RV) and the left ventricle $(\mathrm{LV})$ and the ratio between $\mathrm{RV} / \mathrm{LV}+$ interventricular septum (IVS). ${ }^{18}$

For measuring the thickness of the pulmonary artery, an arbitrary line was drawn from the dorsal to the ventral side on H\&E-stained sections (5 $\mu \mathrm{m}$ thick) using Olympus CellSens software version 7 (Olympus America, Center Valley, PA), and the thickness measured in both the RA and BPD groups in all of the experimental groups was studied.

Quantitative measurements for pulmonary arterial hypertension were done by measuring the ratio of thickness of the arterial wall on H\&E sections and comparing it with the diameter of the arterial lumen (arterial wall thickness/diameter of lumen). For all morphometric analyses, either ImageJ or 
Olympus CellSens software version 7 was used. For measuring the mean alveolar area, the Analyze Particles function of ImageJ was used in conjunction with a custom written macro for the measurement of the lung architecture and alveolar area. Values were pooled for each individual animal for statistical analysis.

\section{PAAT and PAET}

CD1 and C57BL/6 mouse pups at PN14 were anesthetized using a cocktail of xylazine-ketamine. High-resolution transthoracic echocardiography was performed using the Vevo 2100 machine (Visual Sonics, Toronto, ON, Canada) with a high-frequency (18- to 38-MHz) probe (Visual Sonics MS400). A Visual Sonic software analysis tool (Vevo 2100 Imaging System; FujiFilm VisualSonics Inc., Toronto, ON, Canada) was used to obtain pulmonary arterial acceleration time (PAAT) values. A short PAAT or small PAAT/pulmonary arterial ejection time (PAET) ratio indicates high peak systolic pulmonary artery (PA) pressure, as previously described. ${ }^{19}$

\section{Quantitative Real-Time PCR}

The hypothalamus and whole lung samples from mice of both strains from the RA and BPD groups were harvested at PN14 and homogenized, and total RNA was extracted using TRIzol (Invitrogen, Carlsbad, CA) and the Qiagen miRNeasy Kit (Qiagen, Frederick, MD) for synthesis of cDNA. Total RNA (1 $\mu \mathrm{g}$ ) was used for regular cDNA synthesis using the High Capacity cDNA Kit (Applied Biosystems, Foster City, CA), per the manufacturer's instructions, whereas cDNA for miRNA expression was synthesized using the miScript II RT Kit (Qiagen). Real-time PCR was performed in a $20-\mu \mathrm{L}$ volume with SYBR Green (Applied Biosystems) for mRNA quantification and QuantiTect SYBR Green PCR Kit (Qiagen) for miRNA quantification. U6 and $18 \mathrm{~S}$ were used as the reference genes in the lungs, whereas phosphoglycerate kinase 1 ( $P g k l)$ was used as a housekeeping gene for measuring the expression of genes in the brain. ${ }^{20}$ All primers used for real-time quantitative PCR (qPCR) in this study were extracted from the Harvard University primer bank (https://pga.mgh.harvard. edu/primerbank, last accessed January 25, 2019).

\section{miRNA Mimic and Inhibitor Experiments}

Two doses of $20 \mu \mathrm{mol} / \mathrm{L}$ in a volume of $3 \mu \mathrm{L} /$ nostril of miR146 mimic or the inhibitor (Qiagen) were administered intranasally on PN2 and PN4, as described before. ${ }^{17}$ The sex was differentiated on PN2 and PN4, as previously described, ${ }^{21}$ so that males were selectively administered with the mimic and females were administered with the inhibitor, in the same litter.

\section{Western Blot Analysis}

Western blot analyses for glyceraldehyde-3-phosphate dehydrogenase (GAPDH), $\beta$-actin, vinculin, Akt, toll-like receptor 4 (TLR4; Cell Signaling Technology, Danvers, MA; 1:1000), platelet-derived growth factor (PDGF)-A, secreted phosphoprotein 1 (Spp1), IL-1 receptor-associated kinase 1 (IRAK1; Santa Cruz Biotechnology, Dallas, TX; 1:500), and angiopoietin 1 and 2 (Ang1 and Ang2, respectively; 1:500; Sigma, St. Louis, MO) were performed, as previously described, ${ }^{17}$ by loading $30 \mu \mathrm{g}$ of lung protein, followed by immunoblotting with the above antibodies and visualization with Odyssey infrared imaging system (LICOR Biosciences, Lincoln, NE).

\section{miRNA in Situ Hybridization and Immunofluorescence}

In situ hybridization was performed, as previously described, ${ }^{22}$ with modifications for miRNA. Briefly, sections were deparaffinized and dehydrated through a series of graded alcohol and permeabilized with proteinase $\mathrm{K}$ for 15 minutes at $37^{\circ} \mathrm{C}$. A double digoxygenin-labeled locked nucleic acid miR-146-5p probe (40 nm; Qiagen) was incubated on paraffin sections and hybridized overnight at $55^{\circ} \mathrm{C}$. Afterwards, the sections were washed with $5 \times$ saline sodium citrate, $1 \times$ saline sodium citrate, and $0.2 \times$ saline sodium citrate (two times each at $55^{\circ} \mathrm{C}$ for $10 \mathrm{mi}$ nutes) and incubated with anti-digoxygenin antibody (Roche, Nutley, NJ; 1:500) overnight at $4^{\circ} \mathrm{C}$. They were allowed to develop with nitroblue tetrazolium/5-bromo-4chloro-3-indolyl phosphate alkaline phosphatase substrate (Roche) and were subsequently immunostained with SP-C (Abcam, Cambridge, UK; 1:500), RAGE (R\&D Systems, Minneapolis, MN; 1:100), and von Willebrand factor (Dako, Agilent Technologies, Santa Clara, CA; 1:100) for specific colocalization of miR-146 with alveolar type II or type I or endothelial cells, respectively.

\section{Imaging}

All images were captured on an Olympus IX70 with DP73 camera attachment. At least five to seven low-power (magnification, $\times 20$ ) or high-power (magnification, $\times 40$ ) images were acquired for quantification. CellSens software version 7 was used for merging of bright-field with fluorescence images and modified with Adobe Photoshop 13 (Adobe Inc., San Jose, CA) for acquiring the best images.

\section{Statistical Analysis}

All statistical analyses were performed using Graph Pad Prism version 7.0 (GraphPad Software, San Diego, CA). The data are expressed as the means \pm SD with $n=5$ to 7 mice in each group for morphometry, $n=4$ to 5 mice for qPCR analysis, $n=8$ to 10 fields on H\&E sections for image analysis, and $n=5$ to 9 mice for miR-146 mimic/ inhibitor experiments. Groups were compared with the twotailed unpaired $t$-test and one- or two-way analysis of variance, as appropriate. $P<0.05$ was considered statistically significant. 
A

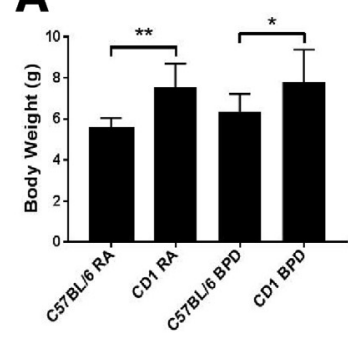

D
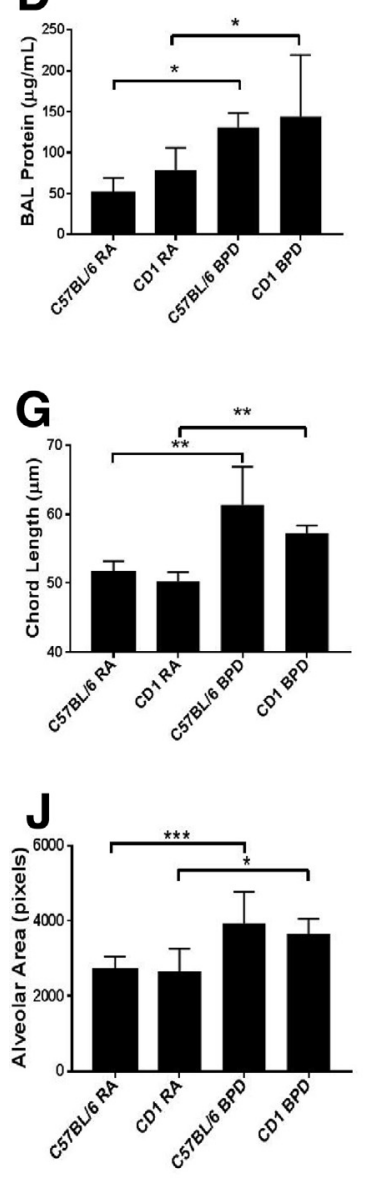

B

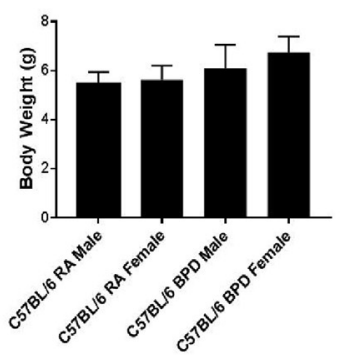

E
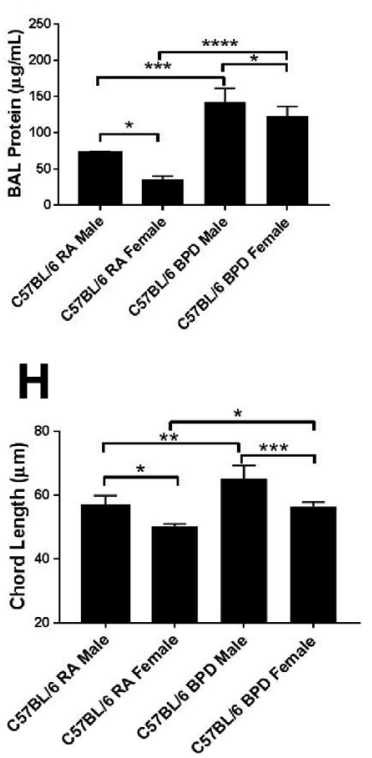

K

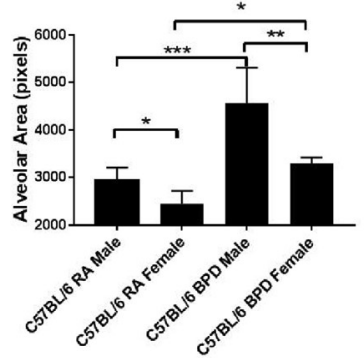

C
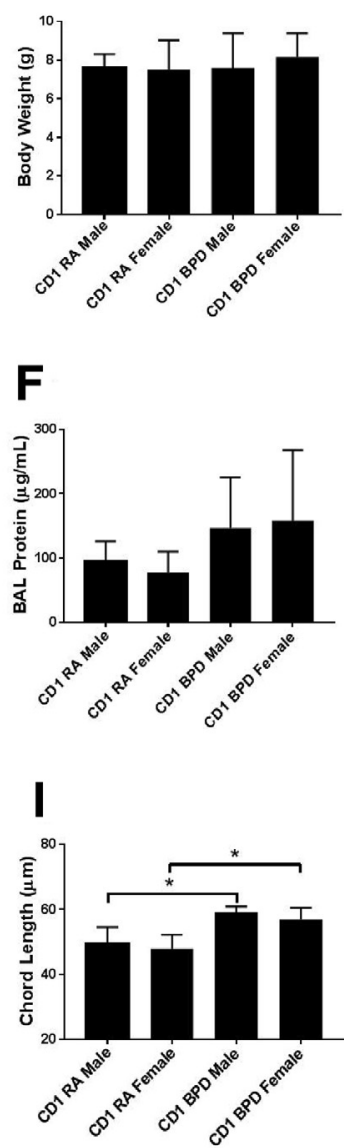

$\mathbf{L}$

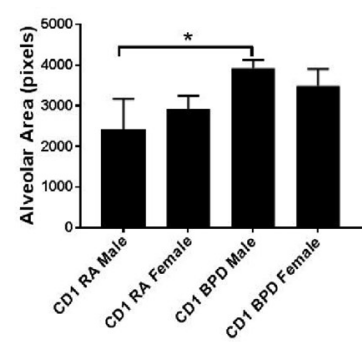

Figure 1 Gross difference between males and females in response to hyperoxia in different strains of mice. A: The average body weight of CD1 mice is significantly higher than that of $\mathrm{C} 57 \mathrm{BL} / 6$ mice in both room air (RA) and bronchopulmonary dysplasia (BPD) groups. B and C: There is no difference in the body weight between $\mathrm{C} 57 \mathrm{BL} / 6$ (B) or CD1 (C) males and females in both RA and BPD groups. D: There is increased bronchoalveolar lavage (BAL) protein in the BPD group of both the strains. E: Males show a significant increase of the protein over females in the $\mathrm{C} 57 \mathrm{BL} / 6$ strain in both RA and BPD groups. F: There is no difference between the two sexes on exposure to hyperoxia in the CD1 strain. G: The chord length is increased in the BPD group of both $\mathrm{C} 57 \mathrm{BL} / 6$ and CD1 strains compared with RA, with no difference between the strains. H: The males have significantly increased chord length over the females in both RA and BPD groups only in the $\mathrm{C} 57 \mathrm{Bl} / 6$ strain. I: In the $\mathrm{CD} 1$ strain, there is no difference between the two sexes in RA; the BPD group, however, shows increased chord length over the RA group. J: The alveolar area is increased in the BPD group of both C57BL/ 6 and CD1 strains compared with RA, with no difference between the strains. K: The males have significantly increased alveolar area over the females in both RA and BPD groups. In BPD males, the alveolar area is significantly higher than in BPD females. In the CD1 strain, the BPD males show increased alveolar area compared with RA males. There is no difference between the males and the females of the BPD group. L: The chord length is increased in the BPD group of both C57BL/ 6 and CD1 strains compared with RA, with no difference between the two strains. The males have significantly increased chord length over the females in both RA and BPD groups only in the C57BL/6 strain. $n=7$ to $8(\mathbf{A}-\mathbf{L}) .{ }^{*} P<0.05$, ${ }^{* *} P<0.01,{ }^{* * *} P<0.001$, and ${ }^{* * *} P<0.0001$.

\section{Results}

Strain and Sex Differences in Response to Severe BPD $\left(100 \% 0_{2}\right)$ with Special Reference to Lung, Heart, and Blood Vessels

To begin trying to elucidate any differences in the two genetic background strains, C57BL/6 mice of the RA and BPD groups were compared with CD1 mice of the RA and BPD groups. Both C57BL/6 and CD1 mice pups were exposed to $100 \% \mathrm{O}_{2}$ to mimic severe BPD in human babies in the neonatal intensive care unit. To assess if there were any changes in morphologic features after hyperoxia exposure, the survival and body weight were recorded in males and females in both the strains; there was no mortality after recovery from severe hyperoxia exposure at PN14 in either males or females of both the strains of mice. As the CD1 pups are larger in size, the BPD group of this strain was expected to have an increased body weight over C57BL/6 BPD pups, even after hyperoxia (Figure 1A). There was no difference in the total body weights in both males and females of the C57BL/6 (Figure 1B) as well as the CD1 strains (Figure 1C).

When examining bronchoalveolar lavage (BAL) protein levels, chord length, and alveolar area, all of the parameters were significantly increased in the pups of the BPD group over the RA group of the same strain. As has been previously reported, ${ }^{5,6,17}$ there was increase in total BAL protein 
A

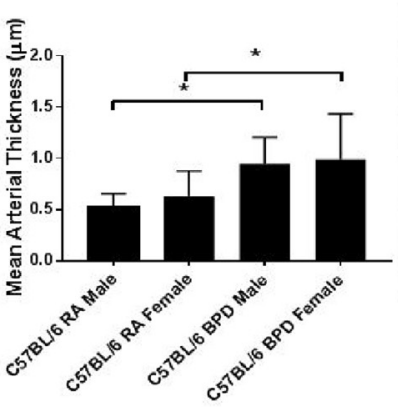

E

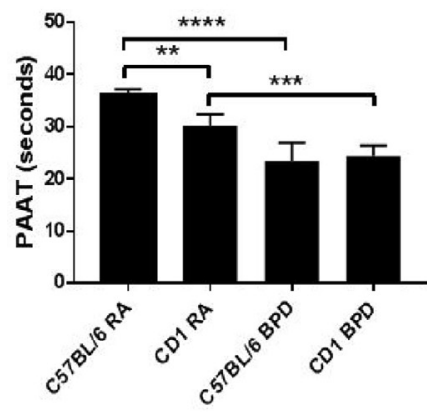

B

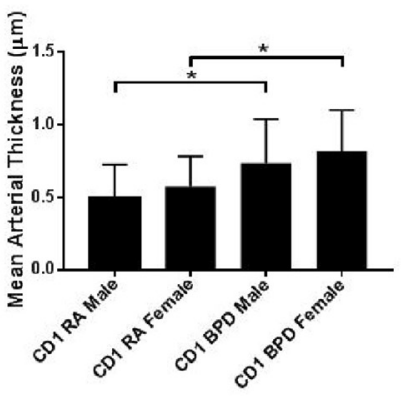

F

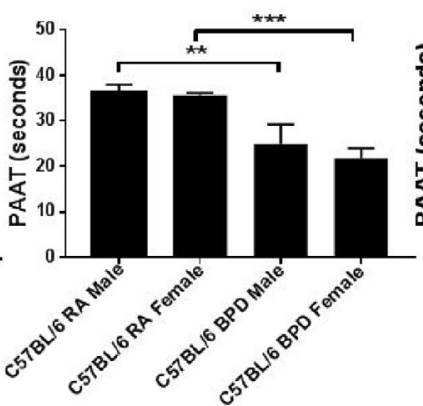

C

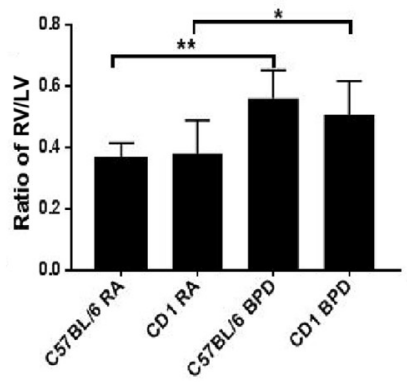

G

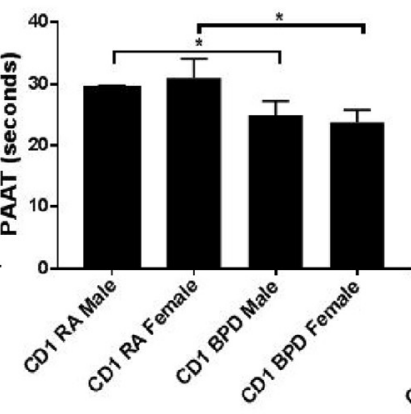

D

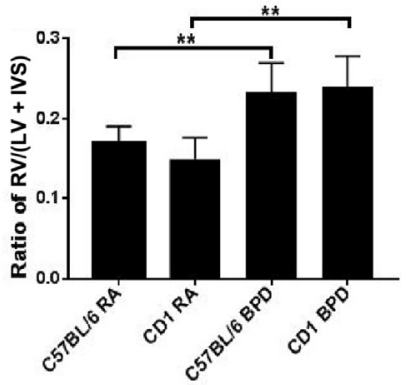

H

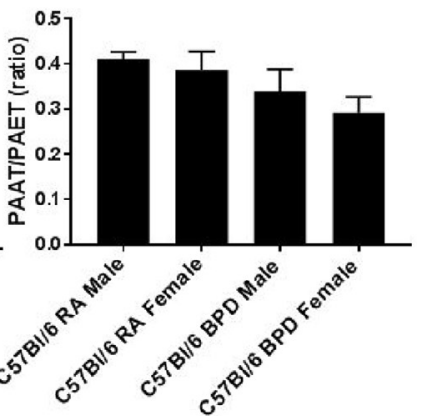

I
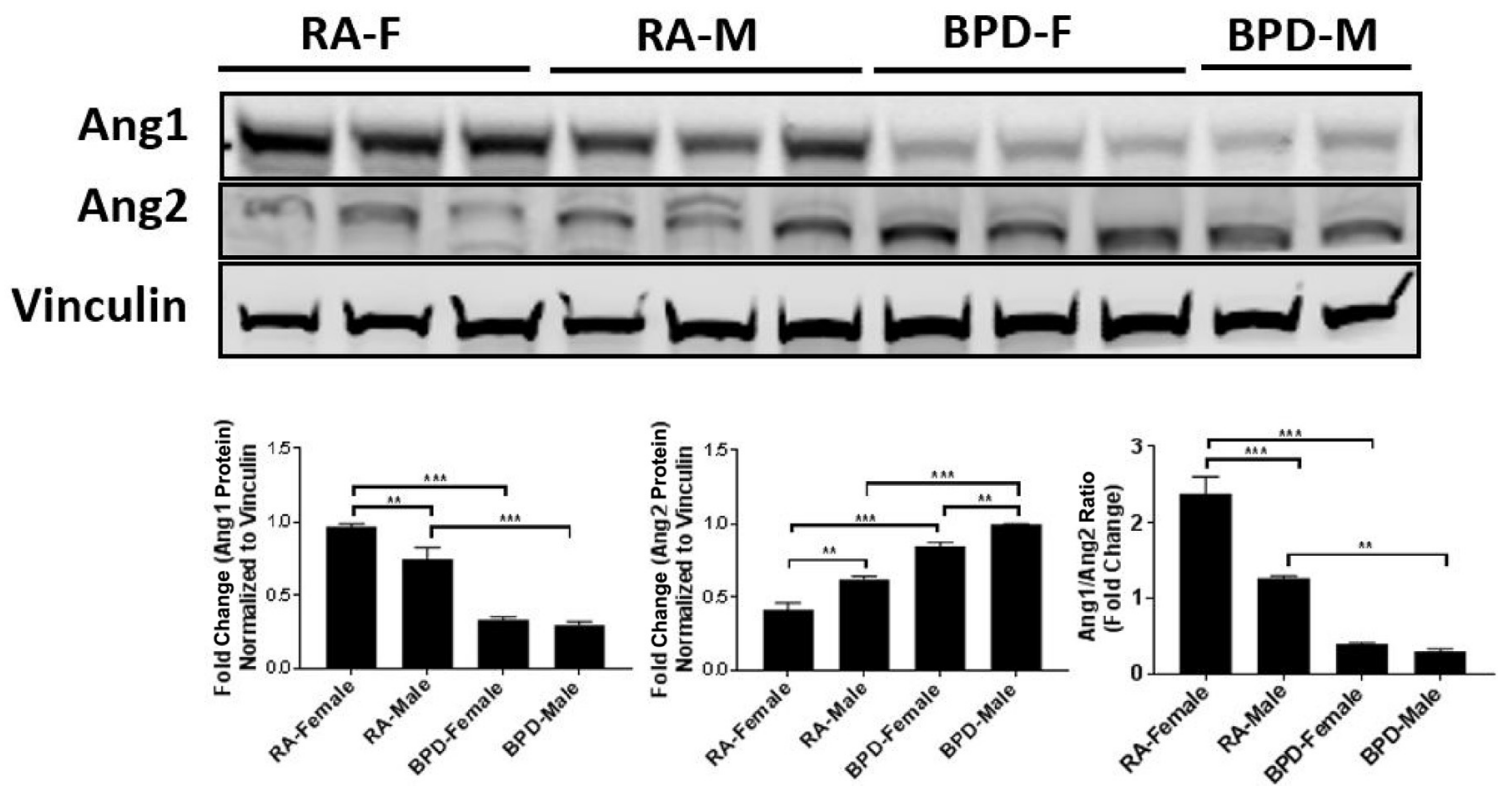

Figure 2 Structural, functional, and hemodynamic changes in the vasculature. A and B: The mean arterial thickness increases in both bronchopulmonary dysplasia (BPD) males (M) and females (F) over room air (RA) controls in both the C57BL/6 (A) and the CD1 (B) strains. C and D: Right ventricular hypertrophy was assessed using right ventricle (RV)/left ventricle (LV) and the Fulton index [RV/LV + interventricular septum (IVS)]. The ratios are higher in the BPD group of the C57BL/6 strain compared with the CD1 strain. E: Both the RA and the BPD groups of the CD1 strain show significantly less pulmonary arterial acceleration time (PAAT) over the $\mathrm{C57BL} / 6$ strain. $\mathbf{F}$ and $\mathbf{G}$ : Both the BPD males and females show decreased PAAT compared with their RA controls in the C57BL/ 6 (F) and the CD1 (G) strains. H: There is no difference in the PAAT/pulmonary arterial ejection time (PAET) ratio in both males and females of RA and BPD groups of both the strains used in this study. I: Representative image of Western blot analysis, showing expression of angiopoietin 1 (Ang1) and Ang2 in males and females of RA and BPD groups (top panel), along with fold-change quantification (bottom panels). Ang1 is higher in females than males, whereas the reverse is true for Ang2 in the RA group. However, Ang2 is higher in BPD males compared with females, whereas there is no change in Ang1 expression between the males and females in the BPD group. $n=7$ to $8(\mathbf{A}-\mathbf{H}) ; n=3(\mathbf{I}) .{ }^{*} P<0.05,{ }^{*} P<0.01,{ }^{*} * P<0.001$, and ${ }^{* * * * P} P 0.0001$. 
A

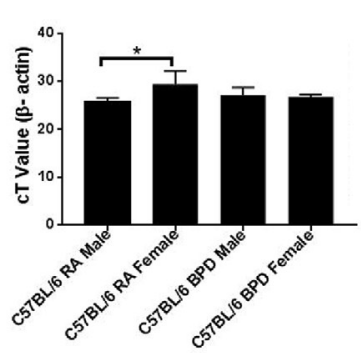

E

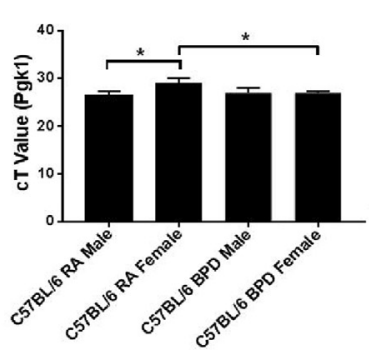

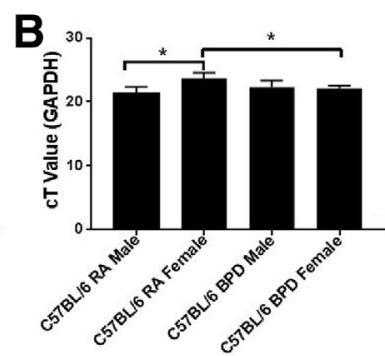

$F$

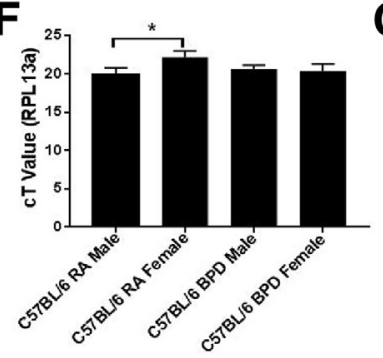

C

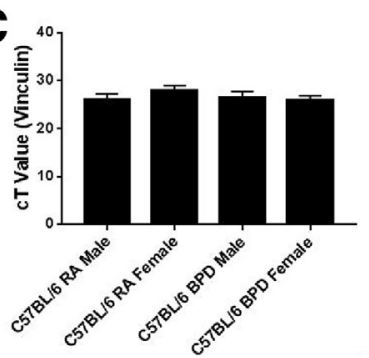

G

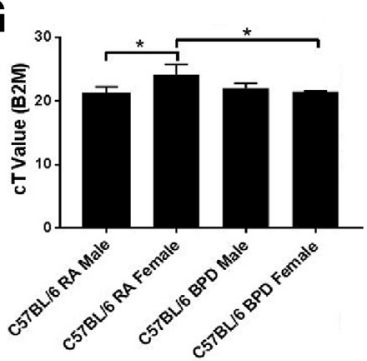

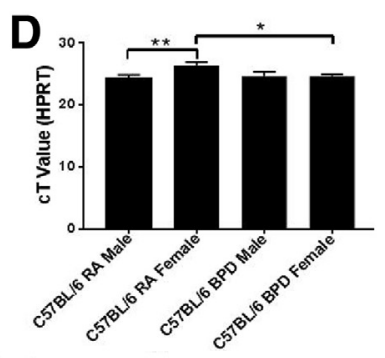

H

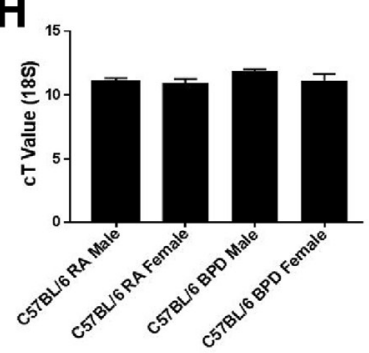

I

Severe BPD
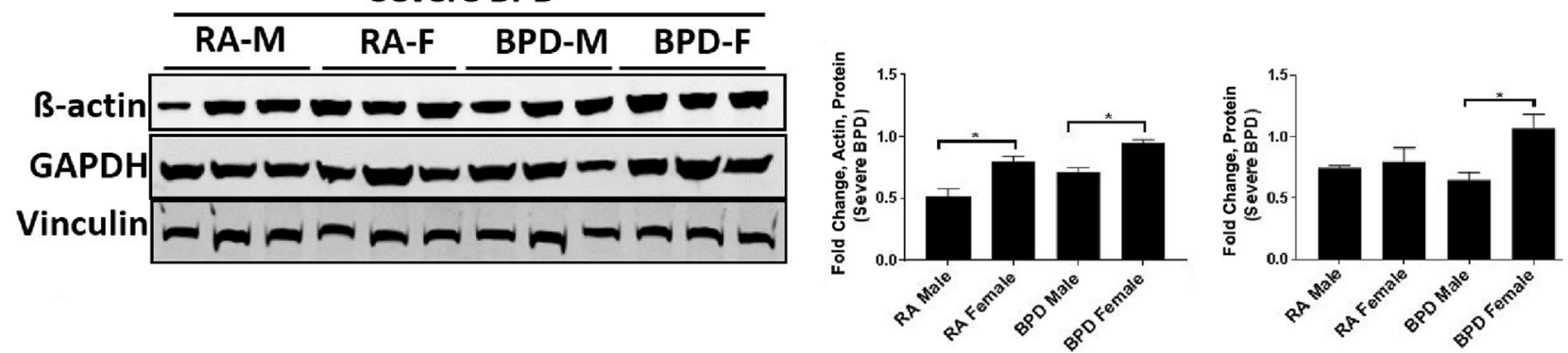

J

Moderate BPD
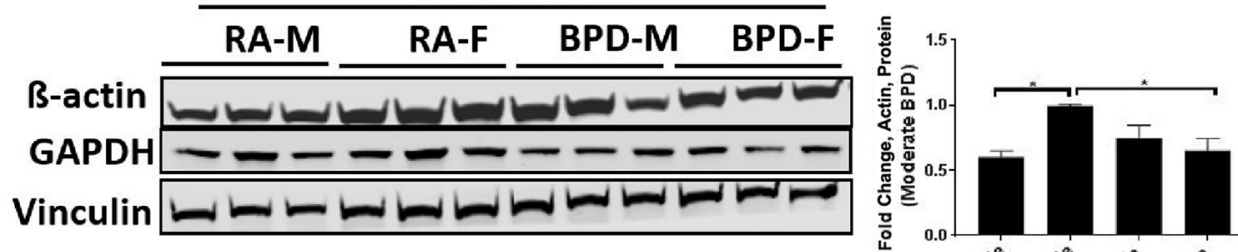

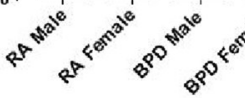

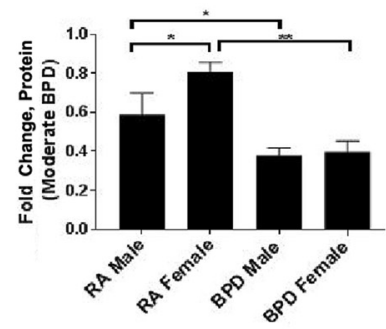

K

Mild BPD
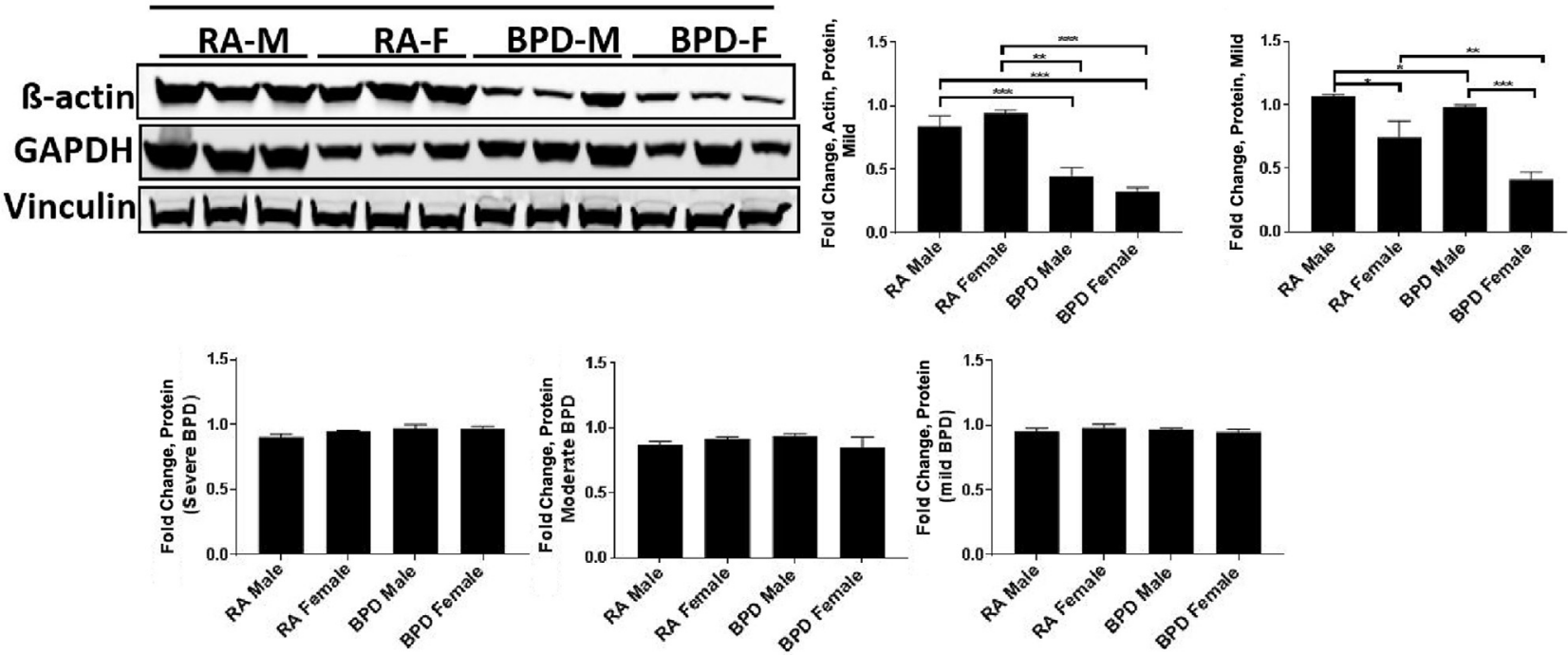
(Figure 1, D-F), chord length (Figure 1, G-I), and alveolar area (Figure 1, J-L) in the BPD groups of both C57BL/6 and CD1 strains. However, no difference was seen in these parameters in the control or in the BPD group of these two different strains. The females showed significantly less BAL protein than males in the C57BL/6 strain (Figure 1E) but not in the CD1 strain (Figure 1F). Both males and females of both strains showed increased BAL protein in the BPD group compared with RA controls (Figure 1, E and F). Chord length is a widely used parameter to characterize the dimensions of distal air space on histologic lung sections. It is a measure of acinar airspace, complex alveoli, and alveolar ducts all combined. The BPD groups of both males and females in both strains had increased chord length compared with RA controls (Figure 1, G-I). Although females showed decreased chord length over males in both RA and BPD groups of the C57BL/6 strain (Figure 1H), there was no significant difference in the chord length between the two sexes in the CD1 strain (Figure 1I). When measuring the alveolar area, it was found that, although there was no difference between the two strains of mice in this study, the BPD groups of both the strains of mice had larger alveolar areas over the RA groups (Figure 1J). The female lungs showed smaller alveolar areas than males in both the RA and the BPD group of the C57BL/6 strain only (Figure $1 \mathrm{~K}$ ) but not the CD1 strain (Figure 1L). Although there was no striking difference between the females of the RA and BPD groups of the CD1 strain, there was an obvious difference in the alveolar area between both the sexes of the C57BL/6 strain; there was only a difference in alveolar area between the males of the CD1 strain in the BPD group over the RA group (Figure 1, K and L). Representative H\&E staining of lung sections from both strains of mice is shown in Supplemental Figure S1A.

The pulmonary artery thickness was measured to assess if there were any changes after hyperoxia treatment in males and females of both C57BL/6 and CD1 strains. There was no significant difference in arterial thickness between the two sexes or between the two strains, although both strains showed increased wall thickness in the BPD group compared with the RA control group (Figure 2, A and B).

As BPD is associated with right ventricular hypertrophy and pulmonary hypertension, ${ }^{23}$ the differences in these parameters in different strains of mice were studied in both males and females. The ratio of RV/LV (Figure 2C) and the Fulton index
(Figure 2D) were higher in the BPD group compared with the RA group in both strains of mice. There was no difference in the RV/LV ratio or the Fulton index in both sexes studied. Differences between the strains became apparent when quantifying PAAT by echocardiography to monitor cardiopulmonary function. PAAT and PAET are quantitative methods used to study the blood flow velocity characteristics in the RV outflow tract in response to changes in ventricular mechanical performance and pulmonary vascular load and compliance. ${ }^{24}$ At baseline, CD1 mice had significantly lower PAAT than C57BL/6 mice, and there was no PAAT and PAET difference between the BPD groups in these two strains (Figure 2E). BPD pups had significantly lower PAAT when compared with RA animals of their respective strains (Figure 2, F and G). However, there was no change in the PAAT/PAET ratio between the RA and the BPD groups (Figure $2 \mathrm{H}$ ).

To assess for differences in the lung vasculature between the two sexes in both the RA and BPD groups in the two strains of mice, von Willebrand factor (a marker for endothelial cells) immunostaining was performed on lung sections of all these groups. There was no difference in the staining between the two sexes in the two strains; the BPD group showed fewer vessels with a disrupted pattern compared with the RA group in both the strains (Supplemental Figure S1B).

Ang1 and Ang2 are the most intensively investigated members of the angiopoietin group. As the endothelium is also affected in BPD, with Ang1 activating Tie2 (a receptor tyrosine kinase expressed on endothelial cells) and Ang2 functioning as a Tie 2 antagonist, the sex-specific expression of these proteins was studied in the BPD system. By competitive binding of Ang1 and Ang2 to Tie2, this interaction promotes vessel assembly and stability. Ang1 was expressed twofold higher in females than males in RA, whereas there was no change in BPD between the two sexes. On the contrary, Ang2 was expressed at least onefold higher in males than females in RA; in the BPD group, there was significantly higher expression of Ang2 in the males compared with females. The Ang1/Ang2 ratio was also 2.2fold higher in females compared with males in RA, with no change in the BPD group (Figure 2I).

\section{Strain and Sex Differences in Response to Mild (40\%) and Moderate $(60 \%)$ BPD}

To assess if mild $\left(40 \% \mathrm{O}_{2}\right)$ and moderate $\left(60 \% \mathrm{O}_{2}\right)$ hyperoxia exposure affects the lungs in a similar way as the severe form of

\footnotetext{
Figure 3 Housekeeping genes as loading controls for bronchopulmonary dysplasia (BPD): Real-time quantitative PCR (qPCR; A-H) and Western blot analyses (I-K) with corresponding quantification bar graphs showing the expression of commonly used housekeeping genes in the C57BL/6 strain. A-C: $\beta$ Actin (A) and glyceraldehyde-3-phosphate dehydrogenase (GAPDH; B) are significantly increased (1.5-fold) in females (F) compared with room air (RA) males $(\mathrm{M})$ in both the RA and the BPD groups, whereas there is no change in vinculin expression (C). D-G: $q P C R$ also shows differences in the expression of hypoxanthine-guanine phosphoribosyl transferase (HPRT; D), phosphoglycerate kinase 1 (Pgk1; E), ribosomal protein L13a (RPL13a; F), and $\beta$-2-microglobulin $(B 2 M ; G)$ in the lungs of both males and females in both RA and BPD groups. H: There is no change in the expression of $18 \mathrm{~S}$ in the lungs. I-K: Similarly, at the protein level, there are significant differences between $\beta$-actin and GAPDH in males and females of RA and BPD groups in all of the BPD experiments [mild (I), moderate $(\mathbf{J})$, and severe $(\mathbf{K})$ ], whereas vinculin remains unchanged $(\mathbf{I}-\mathbf{K})$. Vinculin was used as a loading control for Western blot analyses in lung tissues, whereas $18 \mathrm{~S}$ was used as a reference gene for normalization in $\mathrm{qPCR}$ assays for lungs. $n=8(\mathbf{A}-\mathbf{H}) ; n=3(\mathbf{I}-\mathbf{K}) .{ }^{*} P<0.05,{ }^{* *} P<0.01$, and ${ }^{* * *} P<0.001$. cT, cycle threshold.
} 

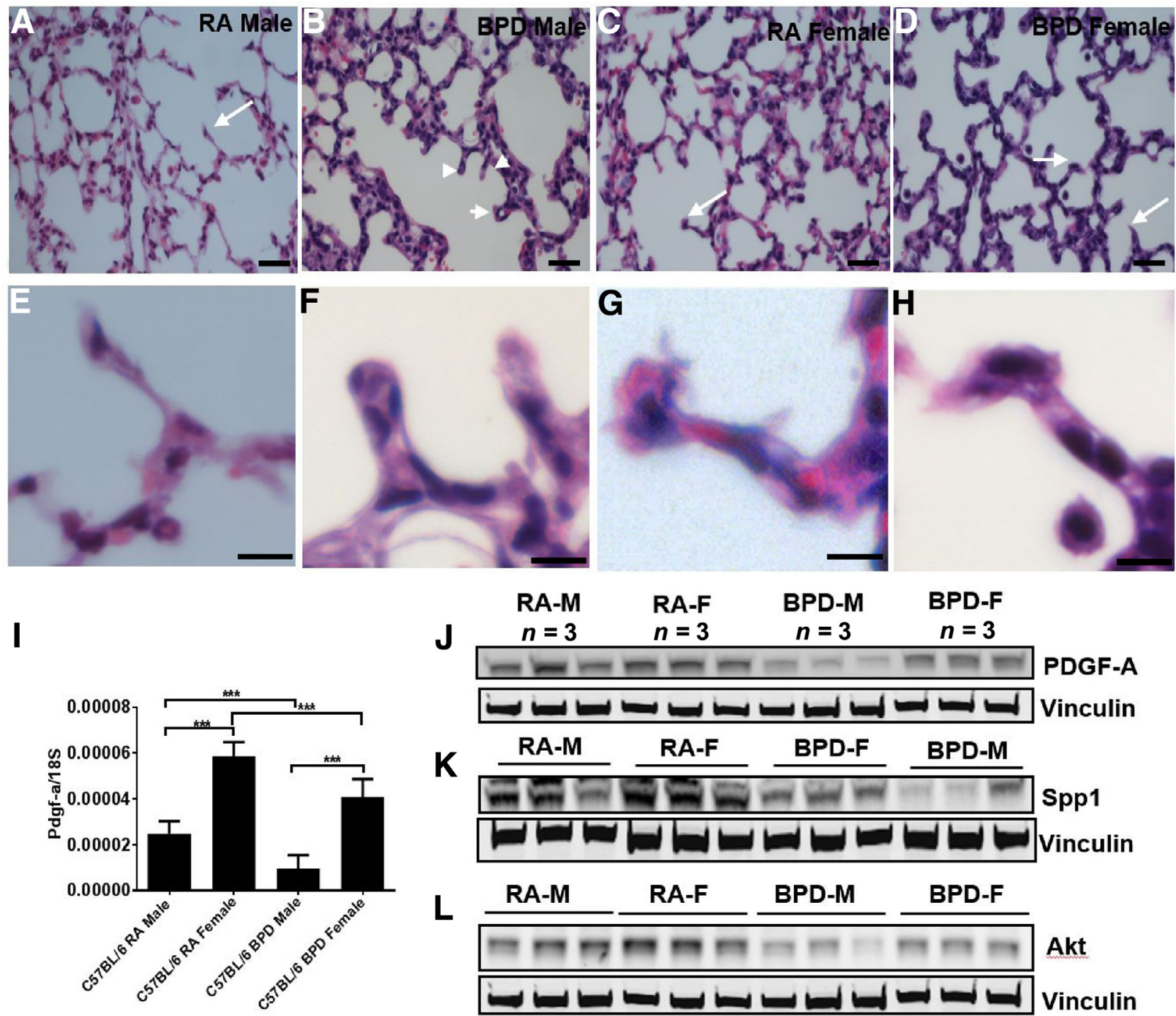

PDGF-A

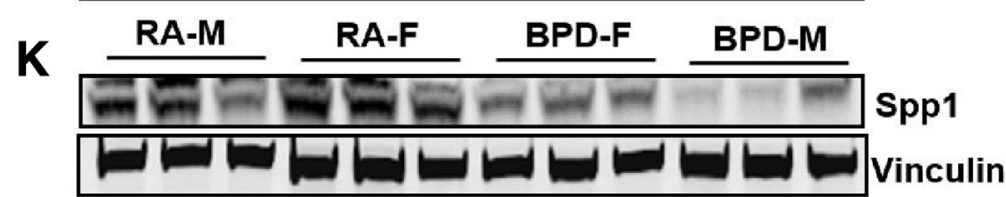

M

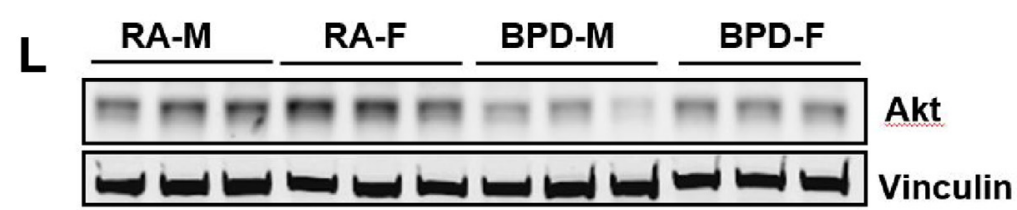

$\mathbf{N}$

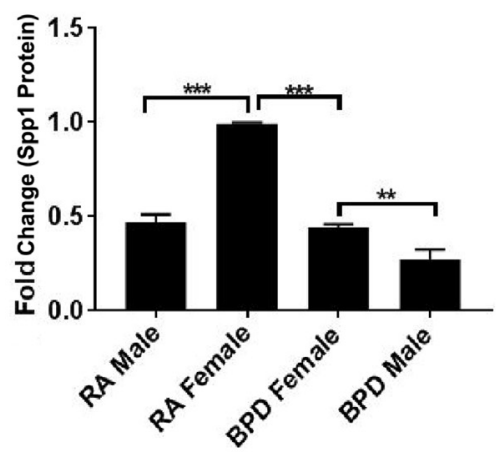

0

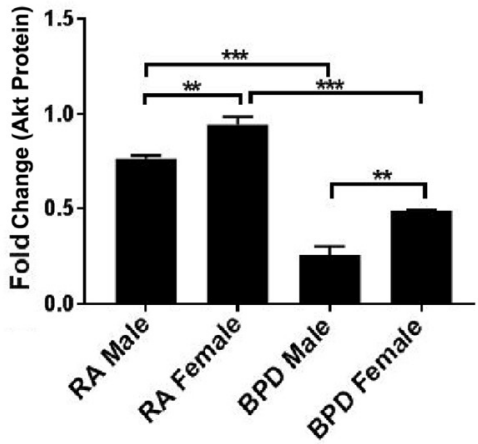

Figure 4 Males (M) and females (F) differ in alveolar septation. A-H: Hematoxylin and eosin staining showing differences in secondary septa during lung development in males and females of the C57BL/6 strain. A-H: Room air (RA) males (A and $\mathbf{E}$ ) show thinner secondary septa than RA females (C and $\mathbf{G}$ ), whereas bronchopulmonary dysplasia (BPD) males (B and F) show short and thick septa with club-shaped ends (arrowheads), compared with BPD females (D and $\mathbf{H}$ ) with pointed tips (arrows). E-H: Higher magnification of the septa tips, indicated with arrows and arrowheads in A-D. Alveolar septation in lung alveogenesis is controlled by genes, such as platelet-derived growth factor-A (PDGFA) and secreted phosphoprotein 1 (Spp1). Akt is considered to play a role in overall lung development. I-0: Real-time quantitative PCR showing expression of PGDF-A (I), along with Western blot analyses of PGDF-A (J), Spp1 (K), and Akt (L), with corresponding densitometric quantifications (M-0). PDGF-A, Spp1, and Akt are expressed more in females than in males in both RA and BPD groups. Vinculin is the loading control. $n=8$ to $10(\mathbf{A}-\mathbf{D}) ; n=3(\mathbf{J}-\mathbf{L}) .{ }^{* *} P<0.01,{ }^{* * *} P<0.001$. Scale bars $=20 \mu \mathrm{m}(\mathbf{A}-\mathbf{H})$. 
injury ( $100 \% \mathrm{O}_{2}$ exposure), PN1 pups from both strains of mice were exposed to $40 \%$ or $60 \% \mathrm{O}_{2}$ for 4 consecutive days and then allowed to recover in RA until P14. In the C57BL/6 strain, BAL protein levels increased in all forms of hyperoxia exposure, and there was no significant difference between $40 \%$, $60 \%$, and $100 \% \mathrm{O}_{2}$ exposure (Supplemental Figure S2A). When evaluating the morphometric parameters (chord length and alveolar area), there was no significant difference between the RA group and the BPD group exposed to $40 \%$, but the $60 \%$ hyperoxia group had increased chord lengths. However, the severe form of BPD showed more injury than the mild form, as assessed by alveolar area (Supplemental Figure S2B) and chord length (Supplemental Figure S2C) in the C57BL/6 strain. These data are in accord with those previously reported by other investigators. ${ }^{14}$

In the CD1 pups, there was no increased BAL protein in the mild and moderate groups (Supplemental Figure S2D). When analyzing morphometrics, significantly increased chord lengths were observed at $40 \%, 60 \%$, and $100 \% \mathrm{O}_{2}$ exposed mice, although significant increases in alveolar area and BAL protein were not found until mice were exposed to $100 \% \mathrm{O}_{2}$ (Supplemental Figure S2, E and F). In terms of morphometric and BAL protein responses to varying severity of $\mathrm{BPD}$, C57BL/6 males and females respond in a fairly similar manner. Both males and females respond in a dose-dependent manner, with increases in chord length, alveolar area, and BAL protein from RA through $100 \% \mathrm{O}_{2}$ (Supplemental Figure S2, G-L). In both males and females, BAL protein seems to be the most sensitive measurement as it increases even at $40 \% \mathrm{O}_{2}$ exposure, whereas chord length and alveolar area need more severe hyperoxia exposure to show changes. As no major differences were found in severe BPD in CD1, C57BL/6 mice were chosen to assess sex-related differences.

\section{Differential Expression of Housekeeping Genes in Males and Females in Response to Mild, Moderate, and Severe BPD}

To understand the mechanism that contributes to a sex-specific difference in BPD, it was important to choose an appropriate reference gene for normalization of all qPCR and Western blot analysis experiments. Hence, several housekeeping genes, including $\beta$-actin $(A C T B)$, hypoxanthine-guanine phosphoribosyl transferase (HPRT), GAPDH, vinculin (VCL), Pgkl, $18 \mathrm{~S}$, ribosomal protein L13a (RPL13A), $\beta$-2-microglobulin $(B 2 M)$, and TATA-box-binding protein $(T B P)$, were tested. There was a marked difference in the expression of all these genes between the males and the females in mild, moderate, as well as severe BPD models (Figure 3, A-K). Vinculin and 18S were the only two housekeeping genes that showed a consistent expression in the lung, without any change between the two sexes and in the RA and BPD groups (Figure 3, C and H-J). Vinculin was chosen as the standard reference loading-control gene for the downstream Western blot studies, whereas $18 \mathrm{~S}$ was used as the normalizing gene in qPCR assays. However, in the pituitary extracts, $\beta$-actin and HPRT could not be used as reference genes for normalization because the females showed more expression of these genes than the males in both RA as well as BPD groups (Supplemental Figure S3, A and B). Pgk1 was the only gene that did not show any variation and was consistent throughout all of the groups in both the sexes exposed to severe hyperoxia (Supplemental Figure S3C).

\section{Expression of GnRH in Hyperoxia-Induced Lung Injury}

Gonadotropin-releasing hormone $(\mathrm{GnRH})$ is the principal regulator for both the trophic hormones (follicle-stimulating hormone and luteinizing hormone); it stimulates secretion of luteinizing hormone, leading to gonadal secretion of the sex steroids testosterone, estrogen, and progesterone. Male sex hormones have been previously shown to increase lung injury in neonatal rats, but no mechanism has since been defined. ${ }^{25}$ Because many morphologic changes were observed in the lungs between males and females in response to hyperoxia compared with RA controls, it was reasoned that the hormones could have a sex-specific influence on lung morphology and physiology. Hence, it was determined if there was any difference in the expression of GnRH that controls the secretion of follicle-stimulating hormone and luteinizing hormone (responsible for sexual dimorphism). Females had similar levels of GnRH as males at RA, whereas the expression of $\mathrm{GnRH}$ in BPD was significantly higher in females than males (Supplemental Figure S4). This may show, to some extent, that $\mathrm{GnRH}$ acts differently in different sexes in response to hyperoxia to exert a protective effect on the female lungs.

\section{Expression of Sex Hormone-Related Genes in Mouse Lung Tissue in Response to Hyperoxia}

In a classic negative feedback loop, sex steroids inhibit secretion of GnRH and also appear to have direct negative effects on gonadotropins. This regulatory loop leads to pulsatile secretion of luteinizing hormone and, to a much lesser extent, follicle-stimulating hormone. Hence, to further explore possible mechanisms behind the sex-related dimorphisms seen in BPD, the expression of various hormone-related genes was examined, including androgen receptor, estrogen receptors (ESTRs) 1 and 2, progesterone receptor, opiate receptor, sex hormone-binding globulin, $\alpha$-fetoprotein, transformationrelated protein 53, and calcitonin gene-related peptide. On qPCR analysis, no significant sex differences were observed in any of the above genes between the RA and BPD groups, but there was a trend toward higher expression of $A R, E S T R 1$, ESTR2, $P R, A F P$, and $C G R P$ in BPD and a trend toward higher expression of $\operatorname{Trp} 53$ in males over females in the BPD group (Supplemental Figure S5).

\section{Biased Expression of Key Genes (PDGFA, Spp1, and Akt) with Respect to Sex in BPD}

Alveolar septation is a key event in alveolarization, during which evaginations arise from within the saccular wall 
A

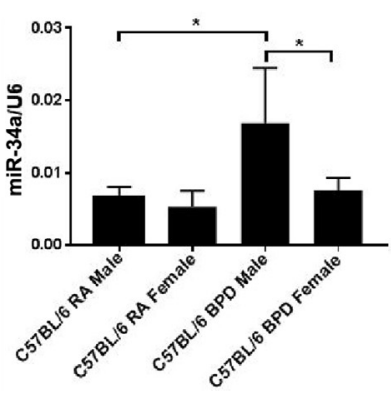

B

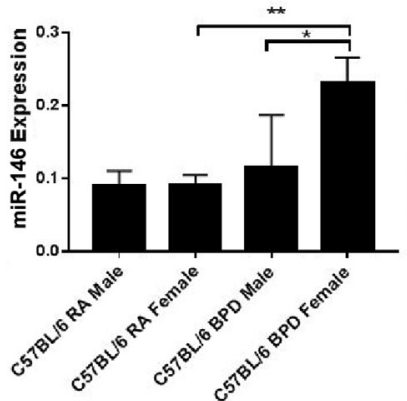

C

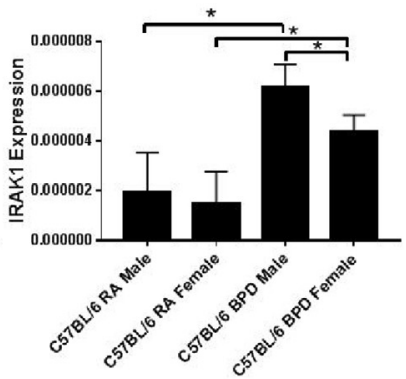

D

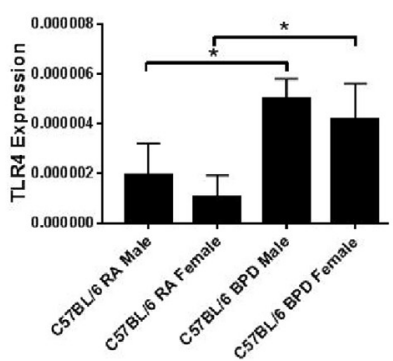

E

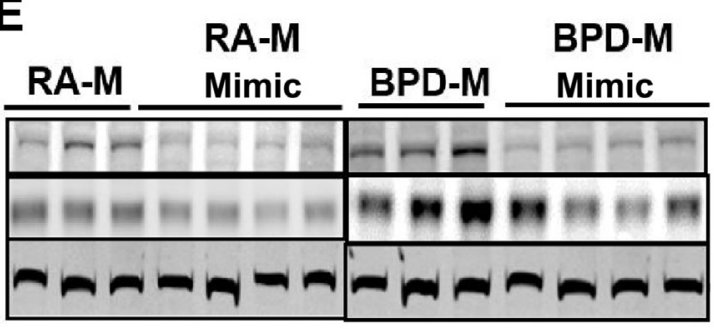

F

RA-F
BPD-F

IRAK-1

TLR4

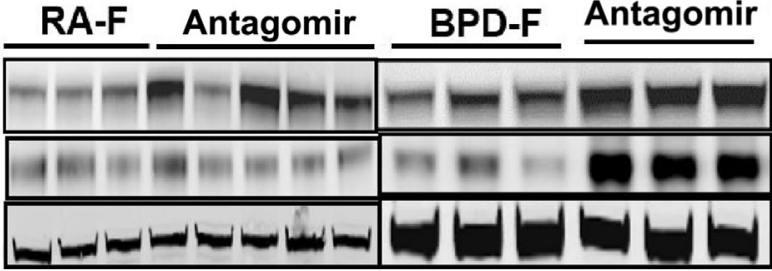

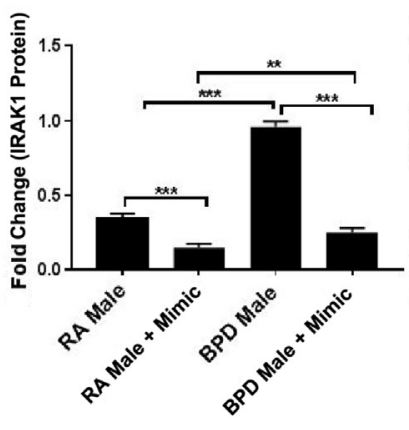

G

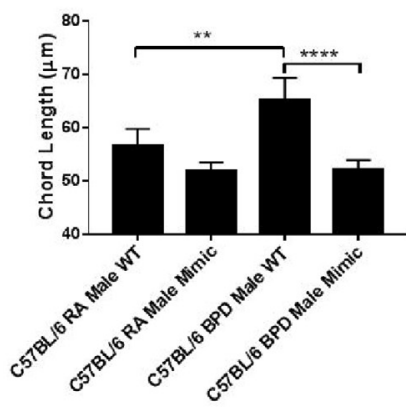

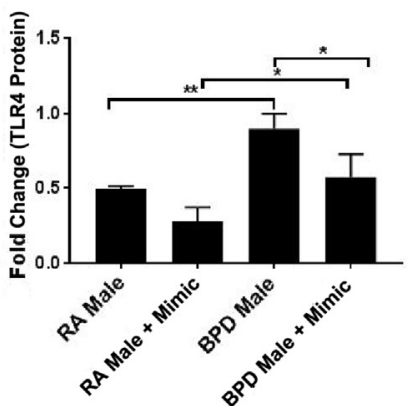

H

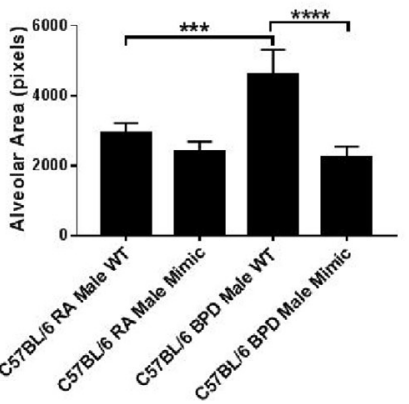

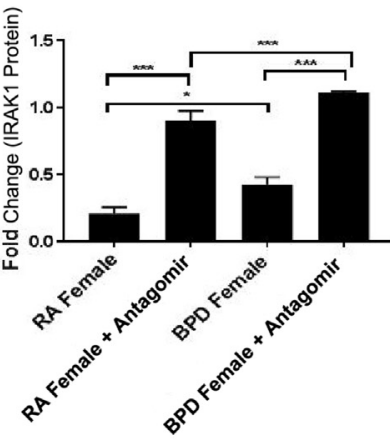

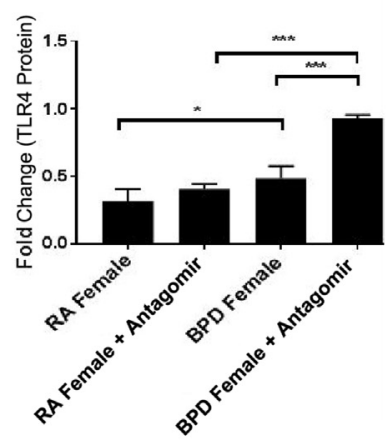

I

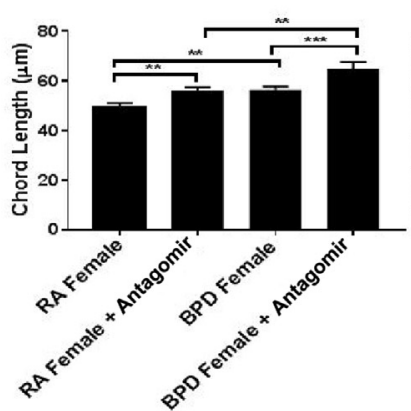

J

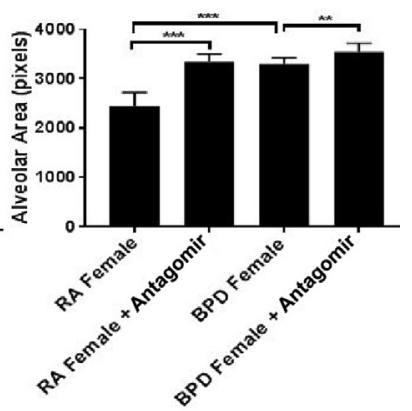

Figure 5 miRNAs (miR-34a and miR-146) are differentially expressed in males (M) and females (F). Real-time quantitative PCR showing expression of miR34a, miR-146, IL-1 receptor-associated kinase 1 (IRAK1), and toll-like receptor 4 (TLR4). A and B: miR-34a is expressed more in bronchopulmonary dysplasia (BPD) males than in BPD females (A), whereas miR-146 is expressed more in the BPD females than males (B). There is no change in the expression of both miRNAs in room air (RA). IRAK1 is a downstream target of miR-146 and is involved in the inflammation pathway. C and D: IRAK1 and TLR4 are significantly upregulated in males and females in the BPD group, but the expression of IRAK1 is significantly decreased in females when compared with males in the BPD group. Lung morphometry showing measurement of chord length and alveolar area in both males and females after treatment with miR-146 mimic and inhibitor, respectively, after exposure to hyperoxia. $\mathbf{E}$ and $\mathbf{F}$ : Both the chord length $(\mathbf{E})$ and the alveolar area (F) are significantly increased in the BPD males compared with RA males, but after treatment with mimic, they are restored to normal. $\mathbf{G}$ and $\mathbf{H}$ : The females given the antagomir show increased chord lengths and alveolar areas compared with wild type (WT). I and J: Representative images of Western blot analyses, with corresponding densitometric quantification showing expression of IRAK1 and TLR4 after treating the males with miR-146 mimic (I) and the females with antagomir (J). The BPD males show increased expression of IRAK1 and TLR4 compared with RA, but after treatment with miR-146 mimic, the expression is significantly decreased. 0n the contrary, in females treated with miR-146 antagomir, IRAK1 and TLR4 are significantly up-regulated in both the RA and BPD groups. Vinculin was used as the loading control. $n=5$ to 8 (A-F). ${ }^{*} P<0.05,{ }^{* *} P<0.01,{ }^{* * *} P<0.001$, and ${ }^{* * * *} P<0.0001$. 
peripheral airways, dividing the distal airspace into alveoli, and thereby leading to a substantial increase in the gas exchange surface of the lung. The whole process is regulated by the concerted action of gene expression programs, growth factor signaling, and extracellular matrix production. ${ }^{26}$ The tips of the secondary septa in females were found to be protruding and seemed to be in an active state of division, whereas the males had blunt tips in the secondary septa. In the BPD group of both the sexes, males had short club-shaped secondary crests with round tips, whereas females had more elongated secondary septa with pointed tips (Figure 4, A-H).

PDGF-A and Spp1 (alias osteopontin) play crucial roles in alveolar septation. Akt is a prosurvival factor, the activation of which preserves alveolar development, whereas its inhibition disrupts normal lung development because of increased apoptosis and results in simplified alveoli, as seen in BPD lungs. ${ }^{27}$ Disruption of the PDGFA gene results in failure of elastic fiber deposition in saccular walls and secondary septation, whereas Sppl promotes pneumocyte growth. Mice lacking Spp1 have smaller, more compliant lungs with an enlarged airspace, similar to the BPD phenotype. ${ }^{28}$ Similarly, Pdgfa knockout mouse lungs have an enlarged alveolar airspace with absence of secondary septation, characteristic of BPD. ${ }^{29}$ Because males have a worse prognosis in BPD than females, it was considered that there would be a differential response in the expression of PDGF-A and Spp1 between males and females. BPD males had a significantly decreased expression of PDGF-A and Spp1 compared with BPD females (Figure 4, $\mathrm{I}-\mathrm{K}, \mathrm{M}$, and $\mathrm{N}$ ), suggesting that lack of adequate secondary septa due to the decreased expression of PDGF-A and SPP1 in males might have contributed to a severe phenotype in BPD. As females did better than males in BPD, more Akt expression was expected in females than males by Western blot analysis. On exposure to hyperoxia, males had a significantly lower expression of Akt compared with females (Figure 4, L and O).

\section{Sex-Specific Expression of miRNAs in Hyperoxia- Induced Lung Injury}

miRNAs were studied to assess if these small regulatory RNAs played any role in mediating this difference in the severe BPD model. Because females do better than males in BPD, males might be might be more susceptible to this condition than females. Also, some miRNAs (eg, miR-146) may regulate innate immunity by interfering in the inflammation pathway. ${ }^{30}$ Using Ingenuity Pathway Analysis, miR-146 and miR-34a were identified to be involved in several inflammatory signaling pathways mediating TLR signaling and triggering receptor expressed on myeloid cells 1 (TREM1) signaling via IRAK1 and Ang1/Ang2 (Supplemental Figure S6). Using TargetScan (http://www.targetscan.org/mmu_72, last accessed December 21, 2018) and miRTarBase (http://mirtarbase.mbc.nctu.edu. tw/php/search.php, last accessed December 21, 2018), several targets that were involved in the pathogenesis of BPD were identified. ${ }^{21,27,31}$ Because miR-34a is a key regulator in $\mathrm{BPD},{ }^{17}$ sex-specific differences in the expression of miR-34a and miR-146 and their downstream targets Ang1 and IRAK1, respectively, were studied. There were no changes in the gene expression of miR-34a and miR-146a between males and females in RA; however, miR-34a was significantly increased in BPD males over BPD females, whereas the converse was true for miR-146, with higher expression in BPD females over BPD males (Figure 5, A and B).

\section{Mediation of Inflammation by miR-146 via the Inflammatory Pathway in Response to Hyperoxia in Males and Females}

Previous studies have shown that inflammation is more prominent in males over females. ${ }^{32}$ As miR-146 is an immediate early-response gene induced by proinflammatory mediators, it was tested if miR-146 had any role in attenuating inflammation in females over males. An increased expression of miR-146a in BPD females was seen over males (Figure 5B). From in situ hybridization and colocalization of in situ hybridization with immunofluorescence, miR-146 was found to be expressed in some type II alveolar epithelial cells, in room air (Supplemental Figure S7). Using RAGE and von Willebrand factor as markers, no expression of miR-146 was seen in type I alveolar epithelial cells or endothelial cells, respectively, in room air (data not shown).

Furthermore, as miR-146 is a negative regulator of TLR4, and IRAK1 is a direct target of miR-146 (downstream of TLR4), ${ }^{30}$ the expression of IRAK1 and TLR4 was studied in both males and females in the severe BPD model. Both TLR4 and IRAK1 were significantly up-regulated in both the sexes in the BPD group, with no differences observed in RA (Figure 5, C and D). In addition, females had significantly lower levels of IRAK1 in BPD compared with males (Figure 5C). These data suggest that IRAK1 levels in BPD females might be suppressed through miR-146 and that suppression of this inflammatory pathway may play a protective role, in part, in females with BPD having better outcomes, compared with males.

To further test that higher expression of miR-146 in females is one of the factors responsible for less damage in the female lungs during BPD, the levels of miR-146 were altered in males and females. miR-146 mimic was administered to the male pups by intranasal administration during hyperoxia exposure, and the males showed a better lung morphology (comparable to BPD females), with increased expression of miR-146, as was evident from BAL protein, total neutrophil count (not shown), and overall lung architecture (chord length and alveolar area). There was no significant difference between the treated BPD males and the males exposed only to RA (Figure 5, E and F). In addition, the females that were administered the miR-146 antagomir showed more damaged lung morphology through increased chord lengths and alveolar areas compared with wild-type animals (Figure 5, G and $\mathrm{H}$ ).

To confirm that miR-146 exerts its effect through inflammation mediated by IRAK1, expression of IRAK1 was 
studied after treatment with miR-146 mimic, followed by hyperoxia. The mRNA levels of IRAK1 were significantly decreased in RA and BPD (Supplemental Figure S8), and the protein levels of IRAK1 and TLR4 were significantly decreased in both RA and BPD animals given the mimic (Figure 5I). IRAK1 levels were significantly increased in the females given the antagomir in RA and BPD groups, whereas TLR4 was significantly increased in the BPD females given the antagomir (Figure 5J), suggesting increased levels of inflammation in the treated females.

\section{Discussion}

Sex is an important biological variable, and investigators have attempted to find a mechanism to explain a preferential diagnosis of a certain disease and its corresponding response to therapy in one sex over the other. Epidemiologic findings of sex differences during lung development indicate a worse male prognosis in several neonatal disorders. ${ }^{33}$ The incidence of BPD is lower among preterm females, and males are often considered an independent predictor for the development of BPD. ${ }^{34}$ Developing therapeutic strategies for BPD has been complicated by a lack of a standardized mouse model used in research laboratories. Between January 2013 and June 2015, 41 different oxygen exposure protocols had been reported in BPD animal models, often resulting in inconsistent findings. ${ }^{35}$ In the present study, we have attempted to find genetic background strain and sex differences in BPD with respect to lung morphology, BPDassociated pulmonary hypertension, select inflammatory pathways involved in BPD, and the importance of sexspecific miRNAs as a contributing factor to BPD. In addition, it was explored if varying concentrations of oxygen impacted BPD in this mouse model.

While comparing the two strains of mice used in this study, CD1 and C57BL/6, it was found that, except for having a significant difference (increase) in the average body weight of the CD1 strain over the C57BL/6 strain, there were no differences between the strains. There was no mortality in either sexes of the two strains at any time point, as has been reported by Lingappan et al. ${ }^{21}$ While evaluating alveolar development between the two strains of mice, there was no difference in the BAL protein, chord length, or alveolar area between the two strains; however, as expected, the BPD group showed increased measurements of the lung injury markers in both strains.

When analyzing male/female differences, although there was no difference in BAL protein, alveolar areas, and chord lengths between the males and females in the CD1 strain, these measurements were all increased in the BPD group over the RA control group. On the contrary, the C57BL/6 males had increased protein in their BAL fluid, chord lengths, and alveolar areas, compared with females, in both the RA and the BPD groups. Thus, males exhibit a more severe lung phenotype than the females in the C57BL/6 strain. Female lungs are smaller and weigh less than those of males and, on average, may contain fewer respiratory bronchioles at birth. Adult female mice and rats have more, but smaller, alveoli than males of the same species, thereby providing them with larger alveolar surface area/body mass ratios. ${ }^{36}$ In humans, the number of alveoli per unit area and alveolar volume do not differ between boys and girls, but boys have larger lungs than girls. Thus, the total number of alveoli and alveolar surface area are larger for boys than for girls for a given age. ${ }^{36}$ Hence, it is not surprising to see larger alveoli in male mice of the BPD group.

Impaired pulmonary angiogenesis, characterized by pulmonary vascular abnormalities, is observed in patients with BPD, which includes dysregulated pulmonary angiogenesis, abnormal pulmonary vascular remodeling, heightened pulmonary vascular tone, and development of abnormal collateral circulations. ${ }^{37}$ In the current study, although there was a striking difference between the number, organization, and patterning of the alveolar endothelial cells between the RA and BPD groups, no major differences in the above vasculature organization were seen between the two sexes in both the strains of mice. A recent study has shown decreased vascularization in female $\mathrm{C} 56 \mathrm{Bl} / 6 \mathrm{~J}$ mice using von Willebrand factor staining after exposure to $95 \% \mathrm{O}_{2}$ from PN1 to PN5 in lung sections at PN21. ${ }^{21}$ Another study that showed sex-specific differences in pulmonary vascularization after neonatal hyperoxia used Sprague-Dawley rats and exposed them to $85 \%$ to $90 \% \mathrm{O}_{2}$ from PN2 to PN $21 .{ }^{38}$ Our study used hyperoxia exposure from PN1 to PN4 and obtained the lung sections at PN14. It is possible that a longer exposure (even an extra day of exposure to the neonatal mouse lung, equivalent to exposing a human preterm lung for 3 to 4 weeks, at this critical developmental stage has a significant impact based on the speed of the developmental changes occurring in the lung at this stage) to hyperoxia-induced injury, waiting until PN21, and the different animal model may have made the differences more obvious. However, significant sex differences were also noted in the expression of angiogenic factors, similar to one group of investigators noted above. ${ }^{21,39}$

Pulmonary hypertension, an abnormal physiological response to a structural alteration in the lung, is often the link between lung dysfunction and the structural and functional adaptation of the right side of the heart. ${ }^{40}$ Pulmonary arterial hypertension results in pulmonary vascular remodeling and shows increased right ventricular systolic pressure and pulmonary vascular resistance that may result in $\mathrm{RV}$ failure. ${ }^{41} \mathrm{To}$ assess pulmonary arterial hypertension in the BPD model, besides PAAT and PAET, other echocardiographic parameters, like PA velocity time integral, PA peak velocity and PA mean velocity, and PA peak gradient and PA mean gradient (data not shown), were also measured. Other than PAAT, no differences were found in all these parameters. BPDassociated pulmonary hypertension may arise because of various insults, including disruption of fetal lung development, perinatal infection/inflammation, mechanical ventilation, and hyperoxia, to cause pulmonary inflammation that 
can have a deleterious impact on airways, alveoli, and pulmonary vasculature. ${ }^{42}$ Increased pulmonary arterial systolic pressure causes early closure of the pulmonary artery and attainment of the peak velocity, therefore decreasing the PAAT.

Herein, we measured the arterial thickness of the pulmonary artery, RV hypertrophy, PAAT, and PAET using echocardiography and found that, although there were significant differences between the BPD and the RA groups in both strains of mice with respect to the above parameters, there were no differences between the males and the females in both RA and BPD groups.

In humans, it is well known that sex differences determine the occurrence of pulmonary arterial hypertension as well as the susceptibility to the treatment. ${ }^{43}$ The survival rate of females was found to be better, establishing the protective effect of the sex-related hormone $17 \beta$ estradiol. ${ }^{43}$ It is also reported that the circulating plasma level of estradiol is similar in both males and females at PN10 in case of rats. ${ }^{44}$ There were no sex-related differences observed in the parameters used to detect pulmonary hypertension in hyperoxiaexposed mice; perhaps a similar estradiol profile until PN14 in both males and females rules out the possibility of a protective effect of estradiol. Oxidation-reduction signaling plays a key role in regulating cell survival in response to hyperoxia. Oxidation-reduction-sensitive pathways are commonly modulated by cysteine thiol modifications by thioredoxin and glutathione oxidoreductase systems. Previous reports have shown that hyperoxia exposure results in impairment of thioredoxin that negatively impacts heat shock protein 90 (HSP90)-oxidative responses to cell survival, leading to impaired lung development and the pathogenesis of BPD. ${ }^{45,46}$ Both males and females likely showed a similar effect in oxidation-reduction signaling modification pathways impacting pulmonary hypertension in response to the hyperoxia conditions.

It is important to remember that, although hyperoxiainduced injury at critical stages of lung development leads to permanent structural and functional impairments (eg, the BPD pulmonary phenotype leads to long-term consequences in the adult ${ }^{47,48}$ ), they may not become apparent during the earlier stages of postnatal life. These assessments were made at PN14; however, maturational changes can modulate the appearance and severity of these changes, which may become more obvious at a later age when reparative processes have maximized or been modulated by differential hormonal levels. Although it is a speculation at this stage, until experimental data (beyond the scope of the present article) are available, the possibility of hormonal or oxidation-reduction signaling effects affecting the phenotype in the long-term cannot be ruled out.

Surprisingly, the loading controls $\beta$-actin, HPRT, GAPDH, Pgk1, RPL13, B2M, and TBP were significantly higher in the lungs of RA females than males. Only $18 \mathrm{~S}$ and vinculin were consistent across the two sexes. Similarly, in the brain tissue, although the females showed increased expression of $\beta$-actin and HPRT at RA over the males, Pgk1 was consistently expressed in both the sexes. All these genes were also differentially expressed across the RA and the BPD groups. Some earlier work ${ }^{49}$ reported that, in the rat liver, there were seven genes that were not constant and were directly or indirectly regulated by sex-dependent hormones, compromising their application as normalizing controls. Similarly, others ${ }^{50}$ have also reported that among 17 housekeeping genes studied in the placentas of males and females, only 3 were stably expressed without any variation between the males and the females. Mild-moderate versus severe hyperoxia exposure led to differential expression of $\beta$-actin and GAPDH. Although actin filaments may contribute to the structural framework of alveolar sacs, GAPDH acts as a sensor during cellular stress and also maintains cytoskeletal architecture and controls cell death. ${ }^{51}$ Prenatally, an effective alveolar-capillary interface is gradually established through a relative thinning of the interstitial compartment by apoptosis. ${ }^{52}$ Postnatally, when apoptosis appears to be involved in trimming off excess type II epithelial cells and fibroblasts to increase lung surface area to enhance gas exchange, an inhibition of apoptosis may contribute to the marked relative increase in epithelial cells. This increase may affect the differential expression of not only $\beta$-actin and GAPDH, but also other genes in the hyperoxia pathway.

Sex hormones influence lung development, and the implications are significant for preterm babies. Although some sex differences are due to classic differences in circulating hormones, there is increasing evidence that genetic factors make an important contribution. ${ }^{53}$ Epidemiologic data suggest the involvement of sex in the incidence, susceptibility, and severity of lung diseases underlying hormonal, physiological, molecular, and developmental differences between males and females. ${ }^{21}$ It is a well-established fact that in many species, surfactant production is delayed in male fetuses compared with the females, ${ }^{54}$ thus emphasizing that hormones might have some role during lung development and maturation. This eventually contributes to its adaptability, to some extent, to withstand or fall prey to any external insult, like hyperoxia-mediated lung injury. In lieu of this, the expression of several hormone-related genes [ $A r$, ESTR1, ESTR2, progesterone receptor ( $P g r), O p r, A f p$, and Trp53] was studied, and no differences in their expression either between males and females or between RA and BPD groups were found at PN14. GnRH had a higher expression in BPD females than males (with no difference at RA). This suggests that a positive feedback loop between the hypothalamus, pituitary, and gonads (ovaries), with increased secretion of estrogen and progesterone in the females, may play a protective role in numerous developmental processes required for functional and physiological adaptation. The above-mentioned hormone-related genes probably do not contribute to the injury caused by BPD in the alveolar stage of lung development in this mouse model.

The lung morphology and the architecture (with respect to chord length, septal thickness, and secondary septa tip) 
were more severely damaged in BPD males than females. As some of the factors that impair lung development after hyperoxia injury are PDGF-A, Spp1, and Akt, ${ }^{28}$ expression of these genes was studied in BPD. PDGF-A is involved in secondary septation, and its absence demonstrates a BPDlike phenotype. The mRNA and protein expression levels of PDGF-A were considerably decreased in BPD males compared with BPD females. Similarly, Akt is a regulatory molecule involved in cell survival, proliferation, and differentiation. Most important, it is a downstream target of phosphatidylinositol 3-kinase, which recruits PDGF-A to exert its action through this pathway. ${ }^{55}$ Akt was also decreased in BPD males compared with females, suggesting that in BPD, PDGF-A is decreased, which further decreases Akt, resulting in larger alveoli, as is the case in males. Spp1 is a functional candidate cytokine for lung development in mice, the absence of which results in larger alveoli, ${ }^{28}$ as is evident in BPD. Herein, BPD males had less Spp1 compared with BPD females. Additional studies are required to evaluate if the above-mentioned genes play a definitive role in structuring the alveolar septa, which is impaired more in the males on hyperoxia exposure.

Recently, miRNAs have been implicated in both early and late lung development. ${ }^{56,57}$ Using Ingenuity Pathway Analysis, in silico approaches, and published literature, it has been shown that miRNAs and their potential targets are involved in BPD. ${ }^{17,58,59}$ We report the differential expression of miR-146 and miR-34a along with their direct targets (IRAK1 for miR-146 and Ang1 for miR-34a) in males and females in BPD. Because there are other targets [sirtuin 1 (Sirt1), Notch1, vascular endothelial growth factor b (Vegfb), B-cell lymphoma 6 (Bcl6), and breast cancer 1 (Brca1) for miR-34a and Irak2, Notch1, nuclear factor kappa-light-chain-enhancer of activated B cells 1 (Nfkb1), wnt1, SMAD4, Wnt5a, and vascular endothelial growth factor a (Vegfa) for miR-146] that are also implicated in BPD, it might be worth analyzing the expression of these candidates in future studies. miR-34a was significantly upregulated and miR-146 was down-regulated in males (with a worse lung phenotype) in BPD. A recovery of the damaged lung with subsequent improvement in its downstream pathways has been reported using an antagomir for miR-34a. ${ }^{17}$ Herein, miR-146 mimic was used to restore the normal lung phenotype in males, and miR-146 antagomir was used to increase alveolar damage in females with exposure to hyperoxia. miR-146 is an NF- $\mathrm{BB}-$ dependent gene, which suggests that it could be actively involved in the inflammation pathway through a negative feedback loop involving down-regulation of IRAK, its direct target. ${ }^{30}$ This begs the question whether IRAK1 was down-regulated with the increase in miR-146a expression (due to miR-146a mimic administration in BPD). IRAK1 was considerably increased in BPD males with respect to females, and miR146 overexpression by miR-146 mimic significantly decreased the IRAK1 levels, which are upstream of IL-1 $\beta$. Blocking miR-146 also significantly increased IRAK1 levels. There was impressive improvement in chord length, BAL protein, alveolar area, and total and neutrophil count exclusively in the males after mimic dosing. Decreased miR-146 may be responsible, in part, for the worse lung phenotype in males after hyperoxia exposure.

To conclude, of the two strains of mice studied, the C57BL/ 6 strain appears to have a more robust BPD phenotype. Severe BPD $\left(100 \% \mathrm{O}_{2}\right)$ caused more damage than moderate BPD $\left(60 \% \mathrm{O}_{2}\right)$, and mild exposure $\left(40 \% \mathrm{O}_{2}\right)$ had a minimal deleterious effect. Vinculin can be used as a loading control for Western blot analysis, whereas $18 \mathrm{~S}$ and Pgk1 can be used as reference genes for qPCR in lung and brain assays, respectively, in BPD research. miRNAs are expressed differentially in males and females; hence, mimics and antagomirs can be selectively used for the two sexes while using them as therapeutic targets. As males have worse prognosis than females, a different dosing regimen should probably be applied for them in treatment strategies. Further detailed structural and functional studies are needed to unravel the sex-specific differences of several molecular signaling pathways involved in BPD.

\section{Acknowledgment}

We thank Dr. Sankar Addya (Thomas Jefferson University, Philadelphia, PA) for the help in Ingenuity Pathway Analysis.

\section{Supplemental Data}

Supplemental material for this article can be found at https://doi.org/10.1016/j.ajpath.2019.01.014.

\section{References}

1. Sigmund CD: Viewpoint: are studies in genetically altered mice out of control? Arterioscler Thromb Vasc Biol 2000, 20:1425-1429

2. Schadt EE, Monks SA, Drake TA, Lusis AJ, Che N, Colinayo V, Ruff TG, Milligan SB, Lamb JR, Cavet G, Linsley PS, Mao M, Stoughton RB, Friend SH: Genetics of gene expression surveyed in maize, mouse and man. Nature 2003, 422:297-302

3. Whitehead GS, Burch LH, Berman KG, Piantadosi CA, Schwartz DA: Genetic basis of murine responses to hyperoxiainduced lung injury. Immunogenetics 2006, 58:793-804

4. Chia R, Achilli F, Festing MFW, Fisher EMC: The origins and uses of mouse outbred stocks. Nat Genet 2005, 37:1181-1186

5. Johnston CJ, Stripp BR, Piedbeouf B, Wright TW, Mango GW, Reed CK, Finkelstein JN: Inflammatory and epithelial responses in mouse strains that differ in sensitivity to hyperoxic injury. Exp Lung Res 1998, 24:189-202

6. Berger J, Bhandari V: Animal models of bronchopulmonary dysplasia: the term mouse models. Am J Physiol Lung Cell Mol Physiol 2014, 307:L936-L947

7. Beery AK, Zucker I: Sex bias in neuroscience and biomedical research. Neurosci Biobehav Rev 2011, 35:565-572

8. Guizzetti M, Davies DL, Egli M, Finn DA, Molina P, Regunathan S, Robinson DL, Sohrabji F: Sex and the lab: an alcohol-focused commentary on the NIH initiative to balance sex in cell and animal studies. Alcohol Clin Exp Res 2016, 40:1182-1191 
9. Clayton JA, Collins FS: Policy: NIH to balance sex in cell and animal studies. Nature 2014, 509:282-283

10. Binet ME, Bujold E, Lefebvre F, Tremblay Y, Piedboeuf B, Canadian Neonatal N: Role of gender in morbidity and mortality of extremely premature neonates. Am J Perinatol 2012, 29:159-166

11. Stevenson DK, Verter J, Fanaroff AA, Oh W, Ehrenkranz RA, Shankaran S, Donovan EF, Wright LL, Lemons JA, Tyson JE, Korones SB, Bauer CR, Stoll BJ, Papile LA: Sex differences in outcomes of very low birthweight infants: the newborn male disadvantage. Arch Dis Child Fetal Neonatal Ed 2000, 83:F182-F185

12. Costeloe K, Hennessy E, Gibson AT, Marlow N, Wilkinson AR: The EPICure study: outcomes to discharge from hospital for infants born at the threshold of viability. Pediatrics 2000, 106:659-671

13. Zakiniaeiz Y, Cosgrove KP, Potenza MN, Mazure CM: Balance of the sexes: addressing sex differences in preclinical research. Yale $\mathrm{J}$ Biol Med 2016, 89:255-259

14. Yee M, Chess PR, McGrath-Morrow SA, Wang Z, Gelein R, Zhou R, Dean DA, Notter RH, O'Reilly MA: Neonatal oxygen adversely affects lung function in adult mice without altering surfactant composition or activity. Am J Physiol Lung Cell Mol Physiol 2009, 297 : L641-L649

15. Maduekwe ET, Buczynski BW, Yee M, Rangasamy T, Stevens TP, Lawrence BP, O'Reilly MA: Cumulative neonatal oxygen exposure predicts response of adult mice infected with influenza A virus. Pediatr Pulmonol 2015, 50:222-230

16. Bhandari V, Choo-Wing $\mathrm{R}$, Lee CG, Yusuf $\mathrm{K}$, Nedrelow JH, Ambalavanan N, Malkus H, Homer RJ, Elias JA: Developmental regulation of NO-mediated VEGF-induced effects in the lung. Am J Respir Cell Mol Biol 2008, 39:420-430

17. Syed M, Das P, Pawar A, Aghai ZH, Kaskinen A, Zhuang ZW, Ambalavanan N, Pryhuber G, Andersson S, Bhandari V: Hyperoxia causes miR-34a-mediated injury via angiopoietin-1 in neonatal lungs. Nat Commun 2017, 8:1173

18. de Visser YP, Walther FJ, Laghmani el H, Steendijk P, Middeldorp M, van der Laarse A, Wagenaar GT: Phosphodiesterase 4 inhibition attenuates persistent heart and lung injury by neonatal hyperoxia in rats. Am J Physiol Lung Cell Mol Physiol 2012, 302: L56-L67

19. Kohut A, Patel N, Singh H: Comprehensive echocardiographic assessment of the right ventricle in murine models. J Cardiovasc Ultrasound 2016, 24:229-238

20. Boda E, Pini A, Hoxha E, Parolisi R, Tempia F: Selection of reference genes for quantitative real-time RT-PCR studies in mouse brain. J Mol Neurosci 2009, 37:238-253

21. Lingappan K, Jiang W, Wang L, Moorthy B: Sex-specific differences in neonatal hyperoxic lung injury. Am J Physiol Lung Cell Mol Physiol 2016, 311:L481-L493

22. Das P, Doyle TJ, Liu D, Kochar J, Kim KH, Rogers MB: Retinoic acid regulation of eye and testis-specific transcripts within a complex locus. Mech Dev 2007, 124:137-145

23. Bhandari A, Bhandari V: Pitfalls, problems, and progress in bronchopulmonary dysplasia. Pediatrics 2009, 123:1562-1573

24. Levy PT, Patel MD, Groh G, Choudhry S, Murphy J, Holland MR, Hamvas A, Grady MR, Singh GK: Pulmonary artery acceleration time provides a reliable estimate of invasive pulmonary hemodynamics in children. J Am Soc Echocardiogr 2016, 29:1056-1065

25. Neriishi K, Frank L: Castration prolongs tolerance of young male rats to pulmonary O2 toxicity. Am J Physiol 1984, 247:R475-R481

26. Pieretti AC, Ahmed AM, Roberts JD Jr, Kelleher CM: A novel in vitro model to study alveologenesis. Am J Respir Cell Mol Biol 2014, 50:459-469

27. Alphonse RS, Vadivel A, Coltan L, Eaton F, Barr AJ, Dyck JR, Thebaud B: Activation of Akt protects alveoli from neonatal oxygen-induced lung injury. Am J Respir Cell Mol Biol 2011, 44: $146-154$

28. Ganguly K, Martin TM, Concel VJ, Upadhyay S, Bein K, Brant KA, George L, Mitra A, Thimraj TA, Fabisiak JP, Vuga LJ, Fattman C,
Kaminski N, Schulz H, Leikauf GD: Secreted phosphoprotein 1 is a determinant of lung function development in mice. Am J Respir Cell Mol Biol 2014, 51:637-651

29. Gouveia L, Betsholtz C, Andrae J: PDGF-A signaling is required for secondary alveolar septation and controls epithelial proliferation in the developing lung. Development 2018, 145. dev161976

30. Taganov KD, Boldin MP, Chang KJ, Baltimore D: NF-kappaBdependent induction of microRNA miR-146, an inhibitor targeted to signaling proteins of innate immune responses. Proc Natl Acad Sci U S A 2006, 103:12481-12486

31. Syed MA, Choo-Wing R, Homer RJ, Bhandari V: Role of nitric oxide isoforms in vascular and alveolar development and lung injury in vascular endothelial growth factor overexpressing neonatal mice lungs. PLoS One 2016, 11:e0147588

32. Cabello N, Mishra V, Sinha U, DiAngelo SL, Chroneos ZC, Ekpa NA, Cooper TK, Caruso CR, Silveyra P: Sex differences in the expression of lung inflammatory mediators in response to ozone. Am J Physiol Lung Cell Mol Physiol 2015, 309:L1150-L1163

33. Gortner L, Shen J, Tutdibi E: Sexual dimorphism of neonatal lung development. Klin Padiatr 2013, 225:64-69

34. Namba F, Ogawa R, Ito M, Watanabe T, Dennery PA, Tamura M: Sex-related differences in long-term pulmonary outcomes of neonatal hyperoxia in mice. Exp Lung Res 2016, 42:57-65

35. Nardiello C, Mizikova I, Silva DM, Ruiz-Camp J, Mayer K, Vadasz I, Herold S, Seeger W, Morty RE: Standardisation of oxygen exposure in the development of mouse models for bronchopulmonary dysplasia. Dis Model Mech 2017, 10:185-196

36. Carey MA, Card JW, Voltz JW, Arbes SJ Jr, Germolec DR, Korach KS, Zeldin DC: It's all about sex: gender, lung development and lung disease. Trends Endocrinol Metab 2007, 18:308-313

37. Alvira CM: Aberrant pulmonary vascular growth and remodeling in bronchopulmonary dysplasia. Front Med (Lausanne) 2016, 3:21

38. Sammour I, Somashekar S, Huang J, Batlahally S, Breton M, Valasaki K, Khan A, Wu S, Young KC: The effect of gender on mesenchymal stem cell (MSC) efficacy in neonatal hyperoxiainduced lung injury. PLoS One 2016, 11:e0164269

39. Coarfa C, Zhang Y, Maity S, Perera DN, Jiang W, Wang L, Couroucli X, Moorthy B, Lingappan K: Sexual dimorphism of the pulmonary transcriptome in neonatal hyperoxic lung injury: identification of angiogenesis as a key pathway. Am J Physiol Lung Cell Mol Physiol 2017, 313:L991-L1005

40. Kumar VHS: Diagnostic approach to pulmonary hypertension in premature neonates. Children (Basel) 2017, 4:75

41. Bossone E, D'Andrea A, D'Alto M, Citro R, Argiento P, Ferrara F, Cittadini A, Rubenfire M, Naeije R: Echocardiography in pulmonary arterial hypertension: from diagnosis to prognosis. J Am Soc Echocardiogr 2013, 26:1-14

42. Bui CB, Pang MA, Sehgal A, Theda C, Lao JC, Berger PJ, Nold MF, Nold-Petry CA: Pulmonary hypertension associated with bronchopulmonary dysplasia in preterm infants. J Reprod Immunol 2017, 124:21-29

43. Mair KM, Johansen AK, Wright AF, Wallace E, MacLean MR: Pulmonary arterial hypertension: basis of sex differences in incidence and treatment response. Br J Pharmacol 2014, 171:567-579

44. Konkle AT, McCarthy MM: Developmental time course of estradiol, testosterone, and dihydrotestosterone levels in discrete regions of male and female rat brain. Endocrinology 2011, 152:223-235

45. Floen MJ, Forred BJ, Bloom EJ, Vitiello PF: Thioredoxin-1 redox signaling regulates cell survival in response to hyperoxia. Free Radic Biol Med 2014, 75:167-177

46. Tipple TE, Welty SE, Nelin LD, Hansen JM, Rogers LK: Alterations of the thioredoxin system by hyperoxia: implications for alveolar development. Am J Respir Cell Mol Biol 2009, 41:612-619

47. Hadouchel A, Delacourt C: Chronic obstructive pulmonary disease following bronchopulmonary dysplasia. Edited by Bhandari V. In Bronchopulmonary Dysplasia. Switzerland: Springer, 2016. pp. 93-108 
48. Landry JS, Banbury SP: Pulmonary function in survivors of bronchopulmonary dysplasia. Edited by Bhandari V. In Bronchopulmonary Dysplasia. Switzerland: Springer, 2016. pp. 281-298

49. Verma AS, Shapiro BH: Sex-dependent expression of seven housekeeping genes in rat liver. J Gastroenterol Hepatol 2006, 21: 1004-1008

50. Cleal JK, Day PL, Hanson MA, Lewis RM: Sex differences in the mRNA levels of housekeeping genes in human placenta. Placenta 2010, 31:556-557

51. Tristan C, Shahani N, Sedlak TW, Sawa A: The diverse functions of GAPDH: views from different subcellular compartments. Cell Signal 2011, 23:317-323

52. Scavo LM, Ertsey R, Chapin CJ, Allen L, Kitterman JA: Apoptosis in the development of rat and human fetal lungs. Am J Respir Cell Mol Biol 1998, 18:21-31

53. Morrow EH: The evolution of sex differences in disease. Biol Sex Differ 2015, 6:5

54. Carey MA, Card JW, Voltz JW, Germolec DR, Korach KS, Zeldin DC: The impact of sex and sex hormones on lung physiology and disease: lessons from animal studies. Am J Physiol Lung Cell Mol Physiol 2007, 293:L272-L278

55. Noskovicova N, Petrek M, Eickelberg O, Heinzelmann K: Plateletderived growth factor signaling in the lung: from lung development and disease to clinical studies. Am J Respir Cell Mol Biol 2015, 52:263-284

56. Bhaskaran M, Wang Y, Zhang H, Weng T, Baviskar P, Guo Y, Gou D, Liu L: MicroRNA-127 modulates fetal lung development. Physiol Genomics 2009, 37:268-278

57. Mujahid S, Logvinenko T, Volpe MV, Nielsen HC: miRNA regulated pathways in late stage murine lung development. BMC Dev Biol 2013, 13:13

58. Ameis D, Khoshgoo N, Iwasiow BM, Snarr P, Keijzer R: MicroRNAs in lung development and disease. Paediatr Respir Rev 2017, 22:38-43

59. Lal CV, Olave N, Travers C, Rezonzew G, Dolma K, Simpson A, Halloran B, Aghai Z, Das P, Sharma N, Xu X, Genschmer K, Russell D, Szul T, Yi N, Blalock JE, Gaggar A, Bhandari V, Ambalavanan N: Exosomal microRNA predicts and protects against severe bronchopulmonary dysplasia in extremely premature infants. JCI Insight 2018, 3:e93994 